\title{
Uniqueness of a quasivariational sweeping process on functions of bounded variation
}

\author{
THOMAS Roche
}

\begin{abstract}
We prove existence and uniqueness of a quasivariational sweeping process on functions of bounded variation thereby generalizing previous results for absolutely continuous functions. It turns out that the size of the discontinuities plays a crucial role: In case they are small enough we prove existence and uniqueness. For large jumps we present a counterexample to the uniqueness of the solution. Finally we show that the condition on the jump size can be replaced by suitable conditions on the shape of the convex set.
\end{abstract}

Mathematics Subject Classification (2010): $49 \mathrm{~J} 40$ (primary); 47J20, 34G25, 34C55 (secondary).

\section{Introduction}

The aim of the present article is to establish existence and uniqueness of a quasivariational (or implicit) sweeping process on functions of bounded variation with small jumps. Here we use the Kurzweil formulation to extend the sweeping process to $B V$. For the proof the input functions are decomposed into parts where the size of the jumps is tiny and a finite number of points where the jumps are allowed to be reasonably large. Both problems are independently solved by a contraction principle.

The sweeping process was introduced by J. J. Moreau [18, 19] and has been intensively studied ever since. The problem reads as follows: Given a time dependent convex set $K(t)$ and an initial value $\xi_{0} \in K(0)$, the aim is to find a function $\xi$ such that

$$
-\dot{\xi}(t) \in \partial I_{K(t)}(\xi(t)) \text { and } \xi(0)=\xi_{0} .
$$

Here $I_{K(t)}$ denotes the indicator function of convex analysis taking value 0 in $K(t)$ and $+\infty$ otherwise. Its subdifferential $\partial I_{K(t)}(x)$ coincides with the normal cone of

Support of the TUM Graduate School Thematic Graduate Center "TopMath" at Technische Universität München, Germany and of the Elite Network of Bavaria through its graduate program "TopMath" is gratefully acknowledged.

Received August 23, 2010; accepted in revised form December 22, 2010. 
$K(t)$ at $x$. Therefore the above inclusion describes the evolution of a point $\xi$ in the following way: If $\xi(t)$ is in the interior of $K(t)$ then it does not move. If it is at the boundary then it moves in the opposite direction of an outer normal vector at this point. To visualize it think of the following: Lay a raisin on a table, place a cake form turned upside down on top of it; how does the raisin move, when the cake form is manoeuvred? The sweeping process is a generalization of this problem, where the cake form can change its shape in time. In fact what we described coincides with an important subclass of this problem where $K(t)=u(t)-Z$ for some given convex set $Z$ and a driving term $u$. This is exactly the play operator from the theory of hysteresis which has been developed from the late 1960s by the Russian school around M. A. Krasnosel'skiı̌ (see e.g. $[4,5]$ ), culminating in the seminal monograph [6]. For an extensive study of the (multidimensional) play operator and its properties we refer to [7] and references therein. Sweeping processes however have been independently studied by a number of people especially M. Monteiro Marques and M. Kunze, see e.g. $[12,17]$. In [13] they introduced the quasivariational sweeping process, which is the subject of our studies here: The convex set $K$ does now no longer depend only on the time $t$ but also on the current state $\xi$ - one can think for example of an elastic cake form (and a rather heavy raisin). The problem then reads

$$
-\dot{\xi}(t) \in \partial I_{K(t, \xi(t))}(\xi(t)) .
$$

The authors derived necessary conditions to prove existence of a solution. The first uniqueness result we are aware of is due to M. Brokate, P. Krejčí and H. Schnabel [2], where sufficient conditions where derived for smooth and bounded convex sets in case the involved functions are absolutely continuous. The proof works by an elaborate fixed point argument. This result was later on generalized by A. Mielke and R. Rossi [15] to unbounded convex sets using the energetic formulation introduced in [16]. Extensions to the space of functions of bounded variation are to be found in $[20,22]$ where by order methods solutions in the sense of differential measure were derived. This notion of solution goes back already to [19]. In particular in these papers no smoothness assumptions on the convex sets was made but a certain monotonicity of the map $t \mapsto K(t, \cdot)$ was required.

For our analysis we adapt the notion of Kurzweil solutions introduced in [9] and expanded later on in [10]. We aim to generalize the result of [2] to functions of bounded variation. However a straightforward transfer of their arguments is not possible: The existence of jumps gives rise to some trouble. In fact, when generalizing the sweeping process from absolutely continuous functions to functions of bounded variation we pay by imposing, additionally to the conditions of [2], a further structural assumption. This is due to the fact that here, because of the existence of jumps, we need to solve static quasivariational inequalities. In the main part of this paper we therefore limit the jump size. However at the end we will also give examples for conditions on the convex sets involved. The idea of the proof is the following: The BV functions are decomposed into intervals where only very small jumps can occur and a finite number of larger jumps. For the first part a method similar to [2] is used to show the existence of a solution. For the larger jumps it 
suffices to analyse the static quasivariational inequality. Here existence and uniqueness can only be guaranteed if the additional structural condition is satisfied, e.g. when the size of the jumps is not too big. The solution to the static problem gives the starting point for the evolution problem on the new time interval. Since we are in $B V$ only a finite number of restarts have to be considered. The framework of the Kurzweil solution is suited to this procedure since at each point of discontinuity of the solution a static quasivariatonal inequality has to be satisfied.

Let us give a short overview of what follows: In the subsequent section we are going to formally introduce the problem and state our main result. Section 3 is devoted to a short repetition of some convex analysis tools needed in the sequel and the analysis of the stationary problem. In Section 4 we introduce the space of functions of bounded variation "with small jumps" and a weighted variation. There we also prove several technical tools required for the main proof, which is the subject of Section 5. It is divided into three parts: First we solve the problem for very small jump sizes, second we construct a solution to our problem and finally we prove its uniqueness. The last section is devoted to a discussion of the jump size condition and structural assumptions on the convex sets. In the appendix we prove a discrete version of Gronwall's lemma and a helpful result on the Kurzweil integral.

ACKNOWLEDGEMENTS. I would like to thank Martin Brokate for insightful discussions and a critical reading of the manuscript.

\section{Statement of the results}

Throughout this work $X$ is a real separable Hilbert space endowed with a scalar product $\langle\cdot, \cdot\rangle$, and norm $|x|=\langle x, x\rangle^{1 / 2}$ for $x \in X$. The evolution process will take place in the space of functions of bounded variation. We additionally impose that some functions are allowed to have small jumps, i.e. discontinuities, only. To be precise let us state the following definition.

Definition 2.1 (BV functions with small jumps). Let $c \geq 0, T>0$. Then we denote by

$$
B V_{L}^{c}(0, T ; \mathcal{Y}):=\left\{f \in B V_{L}(0, T ; \mathcal{Y}): \forall t \in[0, T):|f(t)-f(t+)| \leq c\right\}
$$

the space of all left continuous functions of bounded variation such that the size of every discontinuity is less than $c$.

For a comprehensive introduction of all function spaces used and some tools needed in the sequel we refer to Section 4. We are concerned with the following problem.

Problem 2.2 (Quasivariational sweeping process). Consider a family $Z(r) \subset X$ of closed convex sets parameterized by elements $r$ of a reflexive Banach space $\mathcal{R}$. Assume that $u \in B V_{L}(0, T ; X), g \in B V_{L}\left(0, T ; C^{1}(X \times X ; \mathcal{R})\right)$ and $x_{0} \in$ 
$Z(g(0, u(0), x(0)-u(0)))$ are given. We look for a function $\xi \in B V_{L}(0, T ; X)$ such that

$$
\begin{aligned}
& x(t):=u(t)-\xi(t) \in Z(g(t, u(t), \xi(t))) \quad \forall t \in[0, T], \\
& x(0)=x_{0}, \\
& \int_{0}^{T}\langle x(t+)-y(t), \mathrm{d} \xi(t)\rangle \geq 0 \text { for every } y \in \mathcal{T}(\xi),
\end{aligned}
$$

where

$$
\mathcal{T}(\xi):=\{y \in G(0, T ; X): y(t) \in Z(g(t+, u(t+), \xi(t+))) \forall t \in[0, T]\}
$$

is the set of all admissible testfunctions.

Here $G(0, T ; X)$ denotes the set of all regulated functions mapping $[0, T]$ into $X$, that is all functions $f:[0, T] \rightarrow X$ such that the right- and left hand side limits exists for every $t \in[0, T]$. The integral in (2.4) is supposed to be the Kurzweil (Kurzweil-Henstock) integral introduced in [14], or the Young integral as its special case. This is a generalization of the implicit sweeping process considered in [2] to the space of functions of bounded variation. It also emerges naturally from the sweeping process on $B V$, which reads (in the Kurzweil formulation) as follows:

Problem 2.3 (Sweeping process). For given input functions $u \in B V_{L}(0, T ; X)$, $r \in B V_{L}(0, T ; \mathcal{R})$ and initial condition $x_{0} \in Z(r(0))$, we look for a function $\xi \in B V_{L}(0, T ; X)$ such that

$$
\begin{aligned}
& x(t):=u(t)-\xi(t) \in Z(r(t)) \quad \forall t \in[0, T], \\
& x(0)=x_{0}, \\
& \int_{0}^{T}\langle x(t+)-y(t), \mathrm{d} \xi(t)\rangle \geq 0
\end{aligned}
$$

for every $y \in G(0, T ; X)$ such that $y(t) \in Z(r(t+))$ for every $t \in[0, T]$.

Existence and uniqueness of a solution for this problem have been shown in [10] and local Lipschitz-continuity has been proven recently in [11]. This gives hope that under suitable conditions an existence and uniqueness result for Problem 2.2 can be derived. We denote the norm on $\mathcal{R}$ by $\|\cdot\|$, its dual by $\mathcal{R}^{\prime}$ endowed with the norm $\|\cdot\|_{\mathcal{R}^{\prime}}$ and impose the following assumptions. Note that for any $C \geq 0$ the set $B_{C}(x)$ is the closed ball with radius $C$ centered at $x$, that is $B_{C}(x)=\{y \in$ $X:|y-x| \leq C\}$. Furthermore for a convex set $Z(r)$ with $0 \in Z(r)$ we denote by $M(r, x)$ its Minkowski functional evaluated at a point $x$.

Hypothesis 2.4. There exists $C>0$ such that $0 \in Z(r) \subset B_{C}(0)$ for all $r \in \mathcal{R}$. Furthermore the partial Fréchet derivatives $\partial_{r} M(r, x) \in \mathcal{R}^{\prime}$ and $\partial_{x} M(r, x) \in X$ 
exist for every $r \in \mathcal{R}$ and every $x \in X \backslash\{0\}$. We denote $B(r, x)=\frac{1}{2} M^{2}(r, x)$. The maps

$$
\begin{aligned}
J(r, x) & =\partial_{x} B(r, x)=M(r, x) \partial_{x} M(r, x): X \times \mathcal{R} \rightarrow X, \\
K(r, x) & =\partial_{r} B(r, x)=M(r, x) \partial_{r} M(r, x): X \times \mathcal{R} \rightarrow \mathcal{R}^{\prime}
\end{aligned}
$$

allow continuous extensions to $x=0$. Furthermore, there exist constants $K_{0}, C_{J}$, $C_{K}$ such that for all $x, y \in B_{C}(0), r, s \in \mathcal{R}$ it holds

$$
\begin{aligned}
\|K(r, x)\|_{\mathcal{R}^{\prime}} & \leq K_{0}, \\
|J(r, x)-J(s, y)| & \leq C_{J}\left(|x-y|+\|r-s\|_{\mathcal{R}}\right), \\
\|K(r, x)-K(s, y)\|_{\mathcal{R}^{\prime}} & \leq C_{K}\left(|x-y|+\|r-s\|_{\mathcal{R}}\right) .
\end{aligned}
$$

In [2] it was additionally assumed that all sets $Z(r)$ contain a ball centered at 0 with some radius $c>0$. As has been shown in [11, Lemma 2.3] this is already a consequence of the other assumptions:

Lemma 2.5. If Hypothesis 2.4 hold, then $C_{J} C^{2}>1$ and for $c:=C_{J}^{-1 / 2}$ we have $B_{c}(0) \in Z(r)$ for all $r \in \mathcal{R}$.

Finally let us denote by $C_{\omega, \gamma}^{1}(X \times X ; \mathcal{R})$ the space of functions $f \in C^{1}(X \times$ $X ; \mathcal{R}),(u, \xi) \mapsto f(u, \xi)$ such that

$$
\left\|\partial_{u} f\right\|_{\infty} \leq \omega \wedge\left\|\partial_{\xi} f\right\|_{\infty} \leq \gamma .
$$

Now everything is in place to state our main result.

Theorem 2.6. Let $u \in B V_{L}^{c_{u}}(0, T ; X), g \in B V_{L}^{c_{g}}\left(0,, T ; C_{\omega, \gamma}^{1}(X \times X ; \mathcal{R})\right)$ and $x_{0} \in Z\left(g\left(0, u(0), u(0)-x_{0}\right)\right)$. Assume that Hypothesis 2.4,

$$
\begin{gathered}
\delta:=C K_{0} \gamma<1 \quad \text { and } \\
C K_{0} c_{g}+\left(1+C K_{0} \omega\right) c_{u}<\frac{(1-\delta)^{2}}{C_{J} C(1+\delta)}
\end{gathered}
$$

hold. Then there exists a unique solution to Problem 2.2.

Remark 2.7. The need to impose condition (2.10) is owed to the vectorial nature of the problem. For $X=\mathbb{R}$ it suffices to ensure that (2.9) holds to obtain existence and uniqueness of a solution, see Section 6.2.

To verify that a given problem does indeed satisfy the above assumptions amounts in general to tedious calculations. It has been done for the Gurson model with absolutely continuous functions in [21]. That model, introduced in [3], describes the nucleation of voids in elastic and ideally plastic materials. Here the convex sets are ellipsoids in the space of stress tensors, that is symmetric tensors on $\mathbb{R}^{3}$, namely

$$
Z(r)=\left\{\sigma \in \mathbb{R}_{\mathrm{symm}}^{3 \times 3}: a\|d(\sigma)\|^{2}+r(2 \cosh (b \cdot \operatorname{tr}(\sigma))-1) \leq(1-r)^{2}\right\}
$$

where $\operatorname{tr}(\sigma)$ denotes the trace of $\sigma$ and $d(\sigma)$ the free deviator $d(\sigma)=\sigma-\frac{1}{3} \operatorname{tr}(\sigma) \operatorname{Id}$. 
Indeed the probably most simple sets to which our theorem applies and that do not reduce to the scalar case are ellipsoid in $\mathbb{R}^{2}$.

Example 2.8. Choose $X=\mathbb{R}^{2}$ and $\mathcal{R}=\mathbb{R}$. Furthermore let the set $Z(r)$ be of the form

$$
Z(r)=\left\{x \in \mathbb{R}^{2}: x_{1}^{2}+2 x_{2}^{2} \leq f(r)^{2}\right\}
$$

with $f: \mathbb{R} \rightarrow(0, \infty)$. First we need to ensure that the conditions of Hypothesis 2.4 are satisfied. First we compute $M(x, r)=\left(x_{1}^{2}+2 x_{2}^{2}\right)^{-1 / 2} / f(r)$. To give a concrete example let us assume that

$$
f(r)=\frac{3}{4}+\frac{1}{4} \sin (r)
$$

Then it is easy to see that $Z(r) \subset B_{1}(0)$. Furthermore

$$
\begin{aligned}
& J(x, r)=\left(\frac{3}{4}+\frac{1}{4} \sin (r)\right)^{-1}\left(\begin{array}{c}
x_{1} \\
2 x_{2}
\end{array}\right) \text { and } \\
& K(x, r)=\frac{x_{1}^{2}+2 x_{2}^{2}}{4\left(\frac{3}{4}+\frac{1}{4} \sin (r)\right)^{3}} \cos (r) .
\end{aligned}
$$

It is possible to choose $K_{0}=4, C_{J}=4$ and $C_{K}=28$. Here the choice of $C_{K}$ is rough, however as it does not appear in (2.9) and (2.10) its mere existence suffices. Due to the above estimates we need $\gamma<\frac{1}{4}$ in order to be able to apply our theorem. Assuming that $g$ does only depend on $\xi, e . g$.

$$
g(t, u, \xi)=\gamma \xi_{1}(t)
$$

the jump size of $u$ needs to be bounded by $\frac{(1-4 \gamma)^{2}}{4(1+\gamma)}$.

\section{Preliminaries: convex analysis and quasivariational inequalities}

In this section we are going to set the stage for our subsequent analysis. We are going to shortly recall basic notions from the theory of convex sets and subsequently study static quasivariational inequalities. These preparations will on the one hand provide usefull tools for our examination, on the other hand motivate the conditions employed in the above theorem.

Before we start just a short comment on notation: Throughout this article we are going to employ a notation originating in discrete mathematics, namely denoting for any $n \in \mathbb{N}$ the set $\{1, \ldots, n\}$ by $[n]$. 


\subsection{Convex analysis}

We recall several results established in $[2,7,11]$ on the theory of convex sets and especially projections in Hilbert spaces. For a given convex closed set $Z \subset X$, we define the projection $Q_{Z}: X \rightarrow Z$ onto $Z$ and its complement $P_{Z}=I-Q_{Z}$ ( $I$ is the identity) by the formula

$$
Q_{Z} x \in Z,\left|P_{Z} x\right|=\operatorname{dist}(x, Z):=\inf _{z \in Z}|x-z| \text { for } x \in X .
$$

The projection $Q_{Z}$ has the following properties.

Proposition 3.1. For every $x, y \in X$ we have

(i) $\left\langle P_{Z} x, Q_{Z} x-z\right\rangle \geq 0 \quad \forall z \in Z$,

(ii) $\left\langle P_{Z} x-P_{Z} y, Q_{Z} x-Q_{Z} y\right\rangle \geq 0$,

(iii) $Q_{Z}\left(Q_{Z} x+\lambda P_{Z} x\right)=Q_{Z} x \quad \forall \lambda \geq 0$ and

(iv) $(x \in Z,\langle y, x-z\rangle \geq 0 \forall z \in Z) \Longleftrightarrow\left(x=Q_{Z}(x+y), y=P_{Z}(x+y)\right)$.

Let $Z(r)$ be a family of convex sets parameterized by $r \in \mathcal{R}$. For simplicity let us denote for any two $r, s \in \mathcal{R}$ by $d_{H}(r, s)$ the Hausdorff distance $d_{H}(Z(r), Z(s))$ and by $Q_{r}, P_{r}$ the projection onto $Z(r)$ and its complement respectively. By $n(r, x)$ for $x \in \partial Z(r)$, we denote an element of the normal cone of $Z(r)$ at the point $x$. We are going to rely on [11, Proposition 3.6] which studies the difference between projections onto two sets $Z(r)$ and $Z(s)$.

Proposition 3.2. Assume $Z(r) \subset X$ is nonempty, closed and convex for all $r \in \mathcal{R}$. Furthermere let there exist functions $j: \mathcal{R} \times \mathcal{R} \rightarrow \mathbb{R}$ and $\psi:[0, \infty) \rightarrow[0, \infty)$ such that

$$
\left|n_{r}-n_{s}\right| \leq j(r, s)+\psi(|x-y|)
$$

for all $x \in \partial Z(r), y \in \partial Z(s)$ and $n_{r} \in \partial I_{Z(r)}(x), n_{s} \in \partial I_{Z(s)}(y)$. Then for all $u \in X$ it holds

$$
\left|Q_{r}(u)-Q_{s}(u)\right| \leq d_{H}(r, s)+\min \left\{\left|P_{r}(u)\right|,\left|P_{s}(u)\right|\right\}\left(j(r, s)+\psi\left(d_{H}(r, s)\right)\right) .
$$

The following results can be found in [2, Section 3].

Proposition 3.3. Let Hypothesis 2.4 hold. Then

$$
\begin{aligned}
& d_{H}(r, s) \leq C K_{0}\|r-s\|_{\mathcal{R}} \quad \forall r, s \in \mathcal{R}, \\
& |n(r, x)-n(s, y)| \leq C_{J} C\left(|x-y|+\|r-s\|_{\mathcal{R}}\right) \\
& \forall r, s \in \mathcal{R} \forall x \in \partial Z(r) \forall s \in \partial Z(s) .
\end{aligned}
$$

We relate the two above results in the following way.

Corollary 3.4. Let Hypothesis 2.4 hold. Then for $u \in X$ it holds

$$
\left|Q_{r}(u)-Q_{s}(u)\right| \leq\left(C K_{0}+C_{J} C\left(1+C K_{0}\right)\left|P_{r}(u)\right|\right)\|r-s\|_{\mathcal{R}} .
$$


Proof. Applying (3.5) to Proposition 3.2 we derive

$$
\left|Q_{r}(u)-Q_{s}(u)\right| \leq d_{H}(r, s)+\left|P_{r}(u)\right| C_{J} C\left(d_{H}(r, s)+\|r-s\|_{\mathcal{R}}\right) .
$$

The claim is now obtained by using (3.4).

\subsection{Quasivariational inequalities - the static case}

When trying to analyse a time-dependent problem, it is natural to consider the underlying static problem first - in our case the quasivariational inequality. Apart from that being folklore wisdom we have even more reason to do so: Let $\xi$ be a solution of Problem 2.2 that jumps at some time $\tau \in[0, T)$. Choosing the testfunction $z$ in (2.4) by $z(t)=u(t+)-\xi(t+)$ for $t \neq \tau$ and $z(\tau)=y$ for some $y \in Z(g(\tau+, u(\tau+), \xi(\tau+)))$ leads to

$$
\langle u(\tau+)-\xi(\tau+)-y, \xi(\tau+)-\xi(\tau)\rangle \geq 0 .
$$

Therefore at any time $\tau$ where $\xi$ jumps it has to satisfy a quasivariational inequality. A short study of it will be the subject of this section. Indeed this analysis is crucial in understanding the jump size condition. It was not needed in [2] where the quasivariational sweeping process on absolutely continuous functions was investigated as no jumps could occur.

We shall need the following space of uniformly Lipschitz continuous functions.

$$
\begin{aligned}
\operatorname{Lip}_{\omega, \gamma}(X \times X ; \mathcal{R}):=\{ & f: X \times X \rightarrow \mathcal{R}: \forall(u, \xi),(v, \eta) \in X \times X: \\
& |f(u, \xi)-f(v, \eta)| \leq \omega|u-v|+\gamma|\xi-\eta|\} .
\end{aligned}
$$

We equip $\operatorname{Lip}_{\omega, \gamma}(X \times X ; \mathcal{R})$ with the sup-norm.

For simplicity we will denote by $x$ the term $\xi-u$. Our problem reads:

Problem 3.5. Let $g_{i} \in \operatorname{Lip}_{\omega, \gamma}(X \times X ; \mathcal{R}), i \in\{0,1\}$ and $x_{0} \in Z\left(g_{0}\left(x_{0}, u_{0}\right)\right)$. Let $u_{1} \in X$ be given. We look for $\xi_{1}$ such that

$$
\xi_{1}-u_{1}=Q_{Z\left(g\left(u_{1}, \xi_{1}\right)\right)}\left(x_{0}+\Delta u\right)
$$

where $\Delta f=f_{1}-f_{0}$ for any $f \in\{u, g, \xi, x\}$.

We try to solve this Problem using the Banach contraction principle. For the remainder of this section we set $\xi_{0}:=u_{0}-x_{0}$ and start with a simple proposition.

Proposition 3.6. Assume $x_{0} \in Z(s)$ and $\xi_{1}-u_{1}=Q_{Z(r)}\left(x_{0}+\Delta\right) u$, then

$$
|\Delta \xi| \leq|\Delta u|+d_{H}(r, s) .
$$

Proof. First note that $\Delta \xi=x_{0}+\Delta u-x_{1}$. We therefore estimate

$$
|\Delta \xi| \leq\left|P_{Z(r)}\left(x_{0}+\Delta u\right)\right| \leq\left|P_{Z(r)}\left(x_{0}+\Delta u\right)-P_{Z(r)}\left(x_{0}\right)\right|+\left|P_{Z(r)}\left(x_{0}\right)\right| .
$$

Due to Proposition 3.1 (ii) the first term is less than $|\Delta u|$ and the second is by definition less than $d_{H}(r, s)$. 
Using the above calculation we can estimate the "jump size" $|\Delta \xi|$ of the solution.

Corollary 3.7. If Hypothesis 2.4 holds and $\xi_{1}$ is a solution to Problem 3.5, then

$$
|\Delta \xi| \leq|\Delta u|+C K_{0}(|\Delta g|+\gamma|\Delta \xi|+\omega|\Delta u|) .
$$

Proof. Using 3.6 with $s=g_{0}\left(u_{0}, \xi_{0}\right)$ and $r=g_{1}\left(u_{1}, \xi_{1}\right)$ leads to

$$
|\Delta \xi| \leq|\Delta u|+C K_{0}\|r-s\|_{\mathcal{R}}
$$

It remains to estimate $\|r-s\|_{\mathcal{R}}$, which is done in the usual way $\left\|g_{1}\left(u_{1}, \xi_{1}\right)-g_{0}\left(u_{0}, \xi_{0}\right)\right\|_{\mathcal{R}} \leq\left\|g_{1}\left(u_{1}, \xi_{1}\right)-g_{1}\left(u_{0}, \xi_{0}\right)\right\|_{\mathcal{R}}+\left\|g_{1}\left(u_{0}, \xi_{0}\right)-g_{0}\left(u_{0}, \xi_{0}\right)\right\|_{\mathcal{R}}$ and the proof is complete.

As in [2] we assume that $C K_{0} \gamma=: \delta<1$. This is indeed necessary to obtain uniqueness as the following example demonstrates.

Example 3.8. Let $X=\mathcal{R}=\mathbb{R}$ and $0<c<1<\infty$. Choose

$$
Z(r)=[-1, \max \{\min \{1, r\}, c\}]
$$

and $g_{i}(u, \xi)=u-\xi$ for $i \in[2]$. Then $C=K_{0}=\gamma=1$. For $x_{0}=u_{0}=\xi_{0}=0$ and $u_{1}>1$ any $\xi_{1} \in\left[u_{1}-1, u_{1}-c\right]$ is a solution to the quasivariational inequality.

Proposition 3.9. Let Hypothesis 2.4 and $C K_{0} \gamma=: \delta<1$ hold. If $\xi_{1}$ is a solution to Problem 3.5, then

$$
|\Delta \xi| \leq \frac{1}{1-\delta}\left(C K_{0}|\Delta g|+\left(1+C K_{0} \omega\right)|\Delta u|\right)=: S(|\Delta g|,|\Delta u|) .
$$

\section{Furthermore}

$$
\forall \eta \in \bar{B}_{S(|\Delta g|,|\Delta u|)}\left(x_{0}\right): Q_{Z\left(g\left(\eta, u_{1}\right)\right)}\left(x_{0}+\Delta u\right) \in \bar{B}_{S(|\Delta g|,|\Delta u|)}\left(x_{0}\right) .
$$

Obtaining the assertion is just a simple calculation. Finally we are now able to prove the following

Proposition 3.10. Assume Hypothesis 2.4 holds and $C K_{0} \gamma=: \delta<1$. Furthermore let $|\Delta g|$ and $|\Delta u|$ be chosen small enough such that

$$
C K_{0}|\Delta g|+\left(1+C K_{0} \omega\right)|\Delta u| \leq \frac{(1-\delta)^{2}}{C_{J} C(1+\delta)} .
$$

Then there exists a unique solution $\xi_{1}$ to (3.9) with $x_{1}=u_{1}-\xi_{1} \in \bar{B}_{S(|\Delta g|,|\Delta u|) \text {. }}$ 
Proof. By Proposition 3.2 we have for all $\eta, \xi \in \bar{B}_{S(|\Delta g|,|\Delta u|)}\left(x_{0}\right)$ that

$$
\begin{aligned}
& \left|Q_{Z\left(g\left(\xi, u_{1}\right)\right)}\left(x_{0}+\Delta u\right)-Q_{Z\left(g\left(\eta, u_{1}\right)\right)}\left(x_{0}+\Delta u\right)\right| \\
& \leq\left(C K_{0} \gamma+S(|\Delta g|,|\Delta u|) C_{J} C\left(1+C K_{0} \gamma\right)\right)|\xi-\eta| \\
& \leq\left(\delta+S(|\Delta g|,|\Delta u|) C_{J} C(1+\delta)\right)|\xi-\eta| .
\end{aligned}
$$

Then (3.14) implies that

$$
\delta+S(|\Delta g|,|\Delta u|) C_{J} C(1+\delta)=: \delta^{\prime}<1
$$

and we can apply the Banach contraction principle.

Note that the condition imposed in Proposition 3.10 are exactly the same as those imposed in Theorem 2.6. If they hold, then we denote the solution operator of the quasivariational inequality by

$$
\xi_{1}=: \mathcal{E}\left(\xi_{0}, u_{0}, g_{0}, u_{1}, g_{1}\right) .
$$

\section{Functions of bounded variation}

In this section we very briefly review functions of bounded variations and introduce spaces with uniformly bounded jump size as well as a weighted norm. Throughout this section let $\mathcal{Y}$ be some Banach space endowed with a norm $|\cdot|$. For a real interval $[0, T]$ we define the set of all finite partitions by

$$
\mathcal{D}_{[0, T]}:=\left\{\left(t_{k}\right)_{k=0}^{n} \subset[0, T]: n \in \mathbb{N}, t_{k-1}<t_{k} \forall k \in[n]\right\} .
$$

Then for some function $f:[0, T] \rightarrow \mathcal{Y}$ we define the total variation over a subinterval $[s, t] \subset[0, T]$ by

$$
\operatorname{Var}(f,[s, t]):=\sup \left\{\sum_{k=1}^{n}\left|f\left(t_{k}\right)-f\left(t_{k-1}\right)\right|:\left(t_{k}\right)_{k=0}^{n} \in \mathcal{D}_{[s, t]}\right\} .
$$

when $[s, t]=[0, T]$ we just write $\operatorname{Var}(f)$. The space of functions of bounded variation on $[0, T]$ is defined by

$$
B V(0, T ; \mathcal{Y}):=\{f:[0, T] \rightarrow \mathcal{Y}: \operatorname{Var}(f)<\infty\} .
$$

Equipped with the norm $\|f\|_{B V}:=|f(0)|+\operatorname{Var}(f),\left(B V(0, T ; \mathcal{Y}),\|\cdot\|_{B V}\right)$ is a Banach space. Furthermore we denote by $S(0, T ; \mathcal{Y})$ the set of all step functions, that is functions $f:[0, T] \rightarrow \mathcal{Y}$, for which there exists a partition $\left(t_{n}\right)_{n=0}^{N} \in \mathcal{D}_{[0, T]}$ and elements $f_{n}, \widehat{f}_{n} \in \mathcal{Y}$ for all $n \in[N] \cup\{0\}$ such that

$$
f(t)=\sum_{n=1}^{N} f_{n} \chi_{\left(t_{n-1}, t_{n}\right)}(t)+\sum_{n=0}^{N} \widehat{f}_{n} \chi_{\left\{t_{n}\right\}}(t)
$$


and by $G(0, T ; \mathcal{Y})$ the set of all regulated functions, i.e. functions which allow leftand rightside limits at every point. It is well known that

$$
S(0, T ; \mathcal{Y}) \subset B V(0, T ; \mathcal{Y}) \subset G(0, T ; \mathcal{Y})
$$

when all spaces are equipped with the $\|\cdot\|_{\infty}$-norm and each injection is dense. By $f(t \pm)$ we denote the right/left - side limit of a function $f$ at a given point $\mathrm{t}$, that is

$$
f(t-)=\lim _{h \downarrow 0} f(t-h) \text { and } f(t+)=\lim _{h \downarrow 0} f(t+h) .
$$

The subspace of all left continuous functions, i.e. $f$ such that $f(t)=f(t-)$, within a given function space is marked by the index $L$.

\subsection{Functions of bounded variation with small jumps}

We give an approximation and a closedness result for functions of bounded variation with small jumps. Just shortly recall that

$$
B V_{L}^{c}(0, T ; \mathcal{Y})=\left\{f \in B V_{L}(0, T ; \mathcal{Y}): \forall t \in[0, T):|f(t)-f(t+)| \leq c\right\}
$$

for some $c \geq 0$. Obviously $B V_{L}^{0}(0, T ; \mathcal{Y})=C B V(0, T ; \mathcal{Y})$.

Proposition 4.1 (Closedness of $\left.B V_{L}^{c}\right)$. Let $c \geq 0$ and $\left(u_{n}\right)_{n \in \mathbb{N}} \subset B V_{L}^{c}(0, T ; \mathcal{Y})$ with either

$$
\begin{gathered}
u_{n} \stackrel{\|\cdot\|_{B V} V}{\rightarrow} u \quad \text { or } \\
\operatorname{Var}\left(u_{n}\right) \leq C \text { and } u_{n} \stackrel{\|\cdot\|_{\infty}}{\rightarrow} u
\end{gathered}
$$

then $u \in B V_{L}^{c}$.

Proof. If $u_{n} \stackrel{\|\cdot\|_{B V}}{\rightarrow} u$ then $\operatorname{Var}\left(u_{n}\right)$ is bounded and $u_{n} \stackrel{\|\cdot\|_{\infty}}{\rightarrow} u$. Thus it suffices to prove the second assertion. First of all $u \in B V(0, T ; X)$ since

$$
\operatorname{Var}(u) \leq \liminf _{n \rightarrow \infty} \operatorname{Var}\left(u_{n}\right) \leq C .
$$

Now choose $t \in(0, T]$ arbitrary. For each $\varepsilon>0$ there exists some $\delta_{n}>0$ such that

$$
\forall s \leq t:|t-s| \leq \delta_{n}:\left|u_{n}(t)-u_{n}(s)\right| \leq \frac{\varepsilon}{3} .
$$

Furthermore choose $N \in \mathbb{N}$ large enough such that

$$
\left\|u_{n}-u\right\|_{\infty} \leq \frac{\varepsilon}{3} \forall n \geq N .
$$


Thus for all $s<t$ with $|t-s| \leq \delta_{N}$ we have

$$
|u(t)-u(s)| \leq \varepsilon
$$

and we infer that $u$ is left continuous. It remains to show that the jump size is bounded by $c$. Since $u, u_{n} \in B V_{L}$ we know that $u_{n}$ and $u$ admit a right hand side limits at every point. For $t \in[0, T)$ therefore there exist $\delta, \delta_{n}>0$ such that

$$
\begin{aligned}
\left|u_{n}(t+)-u_{n}(s)\right| & \leq \frac{\varepsilon}{3} \forall s>t:|t-s| \leq \delta_{n} \text { and } \\
|u(t+)-u(s)| & \leq \frac{\varepsilon}{3} \forall s>t:|t-s| \leq \delta .
\end{aligned}
$$

Choose $N$ as above and set $\delta_{n}^{\prime}:=\min \left\{\delta_{n}, \delta\right\}$. Then for all $n \geq N$ and $s>t$ with $|s-t| \leq \delta_{n}^{\prime}$ we have

$$
\left|u(t+)-u_{n}(t+)\right| \leq|u(t+)-u(s)|+\left|u_{n}(t+)-u_{n}(s)\right|+\left|u_{n}(s)-u(s)\right| \leq \varepsilon .
$$

Therefore $u_{n}(t+) \rightarrow u(t+)$ for all $t \in[0, T]$ and we obtain

$$
|u(t)-u(t+)| \leq \liminf _{n \rightarrow \infty}\left|u_{n}(t)-u_{n}(t+)\right| \leq c,
$$

to complete the way.

Lemma 4.2. For any $u \in B V^{c}(0, T ; \mathcal{Y})$ and any $\varepsilon>0$ there exists a partition $\left(t_{n}\right)_{n=0}^{N} \in \mathcal{D}_{[0, T]}$ and values $\left(\widehat{u}_{n}\right)_{n=0}^{N} \subset \mathcal{Y}$ such that for

$$
\widehat{u}(t)=\widehat{u}_{0} \chi_{\{0\}}(t)+\sum_{n=1}^{N} \widehat{u}_{n} \chi_{\left(t_{n-1}, t_{n}\right]}(t)
$$

the following conditions are satisfied:

$$
\begin{gathered}
\|\widehat{u}-u\|_{\infty} \leq \varepsilon, \operatorname{Var}(\widehat{u}) \leq \operatorname{Var}(u) \text { and } \\
\forall t \in[0, T]: \operatorname{Var}(u,[0, t])+\varepsilon \geq \operatorname{Var}(\widehat{u},[0, t]) \geq \operatorname{Var}(u,[0, t])-2 \varepsilon .
\end{gathered}
$$

Proof. The idea of the proof is based on a classical proof by Aumann [1, p. 257f]. It relies on two facts: There exists a sequence $\left(s_{n}\right)_{n=0}^{N_{1}} \in \mathcal{D}_{[0, T]}$ such that

$$
\sum_{n=1}^{N_{1}}\left|u\left(s_{n}\right)-u\left(s_{n-1}\right)\right| \geq \operatorname{Var}(u)-\varepsilon
$$

and for every $u \in B V_{L}(0, T ; \mathcal{Y})$ and for every $\varepsilon>0$ there exists a partition $\left(r_{n}\right)_{n=0}^{N_{2}} \in \mathcal{D}_{[0, T]}$, with $r_{0}=0$ and $r_{N_{2}}=T$ such that

$$
\forall n \in\left[N_{2}\right]: \forall s, t \in\left(r_{n-1}, r_{n}\right]:|u(s)-u(t)| \leq \varepsilon .
$$


The choice of the half-open interval $\left(r_{n-1}, r_{n}\right]$ is due to the left-continuity of $u$. Let $\left(t_{n}\right)_{n=1}^{N} \in \mathcal{D}$ such that $\left\{t_{n}: n \in[N]\right\} \supset\left\{s_{n}: n \in\left[N_{1}\right]\right\} \cup\left\{r_{n}: n \in\left[N_{2}\right]\right\}$ and define

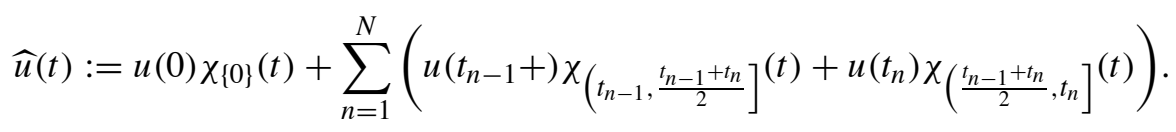

It is easy to see that $\widehat{u} \in B V_{L}^{c / \delta}(0, T ; X)$ and $\|\widehat{u}-u\|_{\infty} \leq \varepsilon$. For each $n \in[N]$ choose a sequence $\tau_{n}^{k} \downarrow t_{n-1}$ as $k \rightarrow \infty$ with $t_{n-1}<\tau_{n}^{k} \leq t_{n}$. Then

$$
\sum_{n=0}^{N}\left(\left|u\left(t_{n-1}\right)-u\left(\tau_{n}^{k}\right)\right|+\left|u\left(\tau_{n}^{k}\right)-u\left(t_{n}\right)\right|\right) \leq \operatorname{Var}(u)
$$

and the lower semicontinuity of the norm gives (4.17). For $t=0$ and $t=T$ (4.18) is straightforward; for the latter use the choice of $s_{n}$. For $t \in(0, T)$ there exists some $n \in[N]$ such that $t \in\left(t_{n-1}, t_{n}\right]$ and we have

$$
\operatorname{Var}(\widehat{u},[0, t])=\sum_{k=1}^{n}\left|u\left(t_{k-1}+\right)-u\left(t_{k-1}\right)\right|+\left|u\left(t_{k-1}+\right)-u\left(t_{k}\right)\right| \leq \operatorname{Var}(u,[0, s])+\varepsilon
$$

for any $s \in\left(t_{n-1}, t\right]$. Here we use $\left|u\left(t_{k-1}+\right)-u\left(t_{k}\right)\right| \leq \varepsilon$ and a limit argument similar as for (4.21). It remains to proof the second inequality. Once again let $t \in(0, T)$ and choose $n \in[N]$ such that $t \in\left(t_{n-1}, t_{n}\right]$. For $t=t_{n}$ we get

$$
\operatorname{Var}\left(\widehat{u},\left[0, t_{n}\right]\right) \geq \operatorname{Var}(u,[0, T])-\varepsilon-\operatorname{Var}\left(\widehat{u},\left[t_{n}, T\right]\right) .
$$

Using $\operatorname{Var}(u,[0, T])=\operatorname{Var}(u,[0, t])+\operatorname{Var}(u,[t, T])$ and the definition of $\widehat{u}$ we see that $\operatorname{Var}\left(\widehat{u},\left[t_{n}, T\right]\right) \leq \operatorname{Var}\left(u,\left[t_{n}, T\right]\right)$. Therefore

$$
\operatorname{Var}\left(\widehat{u},\left[0, t_{n}\right]\right) \geq \operatorname{Var}\left(u,\left[0, t_{n}\right]\right)-\varepsilon .
$$

For $t \in\left(t_{n-1}, t_{n}\right)$ use the above equation,

$$
\operatorname{Var}(\widehat{u},[0, t]) \geq \operatorname{Var}\left(\widehat{u},\left[0, t_{k}\right]\right)-\varepsilon \text { and } \operatorname{Var}(u,[0, t]) \leq \operatorname{Var}\left(u,\left[0, t_{k}\right]\right)
$$

to obtain the desired inequality.

Remark 4.3. As a direct consequence of (4.18) we have that for any $[r, s] \subset[0, T]$

$$
\operatorname{Var}(u,[r, s])+3 \varepsilon \geq \operatorname{Var}(\widehat{u},[r, s]) \geq \operatorname{Var}(u,[r, s])-3 \varepsilon .
$$

Corollary 4.4 (Approximation with stepfunctions). For any $c \geq 0$ and $u \in$ $B V_{L}^{c}(0, T ; \mathcal{Y})$ there exists either a sequence

$$
\begin{aligned}
& \left(u_{n}\right)_{n \in \mathbb{N}} \subset S(0, T ; \mathcal{Y}) \cap B V_{L}^{c}(0, T ; \mathcal{Y}) \text { if } c>0 \text { or } \\
& \left(u_{n}\right)_{n \in \mathbb{N}} \subset S(0, T ; \mathcal{Y}) \cap B V_{L}^{\delta}(0, T ; \mathcal{Y}) \text { if } c=0
\end{aligned}
$$


with $\delta>0$ arbitrary such that

$$
\operatorname{Var}\left(u_{n}\right) \leq \operatorname{Var}(u) \text { and } u_{n} \stackrel{\|\cdot\|_{\infty}}{\rightarrow} u .
$$

In both cases $\left(u_{n}\right)_{n \in \mathbb{N}}$ can be chosen such that $\operatorname{Var}\left(u_{n},[0, t]\right) \rightarrow \operatorname{Var}(u,[0, t])$ uniformly in $[0, T]$.

Proof. For $u \in B V_{L}^{c}(0, T ; X)$ and $n \in \mathbb{N}$ construct $u_{n}$ as in Lemma 4.2 with $\varepsilon=\min \{1 / n, c\}$ if $c>0$ and $\varepsilon=\min \{1 / n, \delta\}$ if $c=0$.

\subsection{A weighted norm on $B V$}

We are going to introduce a norm on $B V$, which is defined in terms of a "weighted total variation".

Definition 4.5 (Weighted total variation). Let $w:[0, T] \rightarrow \mathbb{R}_{>0}$ be monotone decreasing. We call

$$
\operatorname{Var}_{w}(y):=\sup \left\{\sum_{k=1}^{N}\left[\left|y\left(t_{k-1}\right)-y\left(t_{k}\right)\right| w\left(t_{k}\right)\right]:\left(t_{k}\right)_{k=0}^{N} \in \mathcal{D}\right\}
$$

a weighted variation on $[0, T]$ with weight $w$.

One can generalize this definition to positive functions $w$ which are bounded away from zero. However in our subsequent analysis we only employ this notion with monotone decreasing functions. Hence we restrict ourselves to this case and avoid some technical difficulties in the upcoming proofs.

Proposition 4.6. Let $w:[0, T] \rightarrow \mathbb{R}_{>0}$ be monotone decreasing. Then

$$
|y|_{w}:=|y(0)|+\operatorname{Var}_{w}(y),
$$

is a norm on $B V(0, T ; X)$ which is equivalent to $|\cdot|_{B V}$, i.e.

$$
\min \{1, w(T)\}|y|_{B V} \leq|y|_{w} \leq \max \{w(0), 1\}|y|_{B V} .
$$

We call $|\cdot|_{w}$ a weighted norm with weight $w$.

We omit the proof; it is straightforward. Let us point out that any space closed with respect to $|\cdot|_{B V}$ is also closed with respect to $|\cdot|_{w}$. We need the following (lower semi-) continuity results for the dependence of $|\cdot|_{w}$ on its weight.

Proposition 4.7. Let $\left(w_{n}\right)_{n \in \mathbb{N}}, w$ be a (sequence of) monotone decreasing functions

$$
w_{n}, w:[0, T] \rightarrow \mathbb{R}_{>0} .
$$

Assume $w_{n} \rightarrow w$ with respect to $\|\cdot\|_{\infty}$. Then for all $y \in B V(0, T ; \mathcal{Y})$

$$
\operatorname{Var}_{w_{n}}(y) \rightarrow \operatorname{Var}_{w}(y)
$$


Furthermore assume $\left(y_{n}\right)_{n \in \mathbb{N}} \subset B V(0, T ; \mathcal{Y})$ with $\operatorname{Var}\left(y_{n}\right) \leq C$ for some $C$ independent of $n$ and $y_{n} \rightarrow y$ with respect to $\|\cdot\|_{\infty}$, then

$$
\operatorname{Var}_{w}(y) \leq \liminf _{n \rightarrow \infty} \operatorname{Var}_{w_{n}}\left(y_{n}\right) .
$$

Proof. Let $y \in B V(0, T ; \mathcal{Y})$. Note that

$$
\begin{aligned}
& \operatorname{Var}(y)\left\|w_{n}-w\right\|_{\infty} \\
& \geq \sum_{k=1}^{N}\left[\left|y\left(t_{k-1}\right)-y\left(t_{k}\right)\right| w_{n}\left(t_{k}\right)\right]-\sum_{k=1}^{N}\left[\left|y\left(t_{k-1}\right)-y\left(t_{k}\right)\right| w\left(t_{k}\right)\right]
\end{aligned}
$$

for all $\left(t_{k}\right) \in \mathcal{D}$. Thus

$$
\operatorname{Var}(y)\left\|w_{n}-w\right\|_{\infty} \geq \operatorname{Var}_{w_{n}}(y)-\operatorname{Var}_{w}(y)
$$

and by the same method we derive

$$
-\operatorname{Var}(y)\left\|w_{n}-w\right\|_{\infty} \leq \operatorname{Var}_{w_{n}}(y)-\operatorname{Var}_{w}(y) .
$$

Both together imply the first statement via

$$
\left|\operatorname{Var}_{w_{n}}(y)-\operatorname{Var}_{w}(y)\right| \leq \operatorname{Var}(y)\left\|w_{n}-w\right\|_{\infty} .
$$

For the second claim notice that $\operatorname{Var}_{w}$ is lower semicontinuous with respect to the $\|\cdot\|_{\infty}$. Therefore we have

$$
\liminf _{n \rightarrow \infty} \operatorname{Var}_{w_{n}}\left(y_{n}\right) \geq \liminf _{n \rightarrow \infty}\left(\operatorname{Var}_{w}\left(y_{n}\right)-C\left\|w_{n}-w\right\|_{\infty}\right) \geq \operatorname{Var}_{w}(y) .
$$

\section{Proof of the main result}

In this section we prove Theorem 2.6: Assume that Hypothesis 2.4 holds then there exists a unique solution of the quasivariational sweeping process as long as (2.9) and (2.10) are satisfied. For convenience we shall divide the proof into two parts first we establish the existence of a solution in Proposition 5.5 and then we show its uniqueness in Proposition 5.7. First, however, we prove the existence of a unique solution in case the involved functions have very small jumps only.

\subsection{Existence and uniqueness for functions with (very) small jumps}

The idea of the proof is essentially the same as in [2]. However since $B V$ functions are involved an approximation as in [11] is needed. Therefore the proof turns out to be quite technical and quite lengthy. We start by considering the sweeping process 
(Problem 2.3). Our aim is to employ Banach's contraction principle. As mentioned in Section 2, in [10] it was proved that Problem 2.3 has a unique solution $\xi \in$ $B V_{L}(0, T ; X)$ for every $u \in B V_{L}(0, T ; X)$ and every $r \in B V_{L}(0, T ; \mathcal{R})$. We start by considering the case where $u^{i}, r^{i}, i \in[2]$ are step functions on the same division $\left(t_{k}\right)_{k=0}^{n} \in \mathcal{D}_{[0, T]}$. More specifically, let elements $u_{k}^{i} \in X, r_{k}^{i} \in \mathcal{R}$ for $k \in[n] \cup\{0\}$ be given and

$$
f(t)=f_{0} \chi_{\{0\}}(t)+\sum_{k=1}^{n} f_{k} \chi_{\left(t_{k-1}, t_{k}\right]}(t),
$$

where $f$ stands for $u^{i}, r^{i}, i \in[2]$. The solutions $\xi^{i}, x^{i}$ corresponding to $u^{i}, r^{i}$ and initial condition $x_{0}^{i}$ are given by formula (5.1) as well, with $f$ replaced successively by $\xi^{i}, x^{i}$ and $y$, where $\xi_{0}^{i}=u_{0}-x_{0}^{i}$, and

$$
x_{k}^{i}=Q_{r_{k}^{i}}\left(x_{k-1}^{i}+\Delta_{k} u^{i}\right), \quad \xi_{k}^{i}=\xi_{k-1}^{i}+P_{r_{k}^{i}}\left(u_{k}^{i}-\xi_{k-1}^{i}\right),
$$

for $i \in[2], k \in[n]$, where we denote $\Delta_{k} f:=f_{k}-f_{k-1}$ for all $k \in[n]$.

We start with the following result proven in [11, Corollary 5.3].

Lemma 5.1. Assume that Hypothesis 2.4 holds, let $c$ be as derived in Proposition 2.5 and denote

$$
V_{k}:=\left\|\Delta_{k} r^{1}\right\|_{\mathcal{R}}+\left\|\Delta_{k} r^{2}\right\|_{\mathcal{R}}+\left|\Delta_{k} u^{1}\right|+\left|\Delta_{k} u^{2}\right| .
$$

Then there exist constants $\alpha_{1}, \alpha_{2}$ depending only on $C, C_{K}, C_{J}, K_{0}$ such that for every $k \in[n]$

$$
\begin{aligned}
& \left|\Delta_{k}\left(\xi^{1}-\xi^{2}\right)\right|+C \Delta_{k}\left|B\left(r^{1}, x^{1}\right)-B\left(r^{2}, x^{2}\right)\right| \\
& \leq C K_{0}\left\|\Delta_{k}\left(r^{1}-r^{2}\right)\right\|_{\mathcal{R}}+\frac{C}{c}\left|\Delta_{k}\left(u^{1}-u^{2}\right)\right|+\alpha_{1} V_{k}\left|x_{k-1}^{1}-x_{k-1}^{2}\right| \\
& \quad+\alpha_{2} V_{k}\left(\max _{i \in\{k, k-1\}}\left|u_{i}^{1}-u_{i}^{2}\right|+\left(1+V_{k}\right) \max _{i \in\{k, k-1\}}\left\|r_{i}^{1}-r_{i}^{2}\right\|_{\mathcal{R}}\right) .
\end{aligned}
$$

Now assume that there exist stepfunctions $\eta^{i} \in S(0, T ; X), i \in[2]$ and $g \in$ $S\left(0, T ; C_{\omega, \gamma}^{1}(X \times X ; \mathcal{R})\right)$ such that they can be written in the form of (5.1). Let $u^{1}=u^{2}=: u$ and $x_{0}^{1}=x_{0}^{2}=: x_{0}$ and set

$$
r^{i}:[0, T] \rightarrow \mathcal{R}, t \mapsto g\left(t, u(t), \eta^{i}(t)\right) .
$$

Then both $r^{i}$,s are still step functions which can be denoted in the fashion of (5.1). Our intention is to use the estimate of Lemma 5.1 to employ a Gronwall-like estimate (Lemma A.1). However still some preparations are needed. 
Corollary 5.2. Assume that Hypothesis 2.4 holds, let $c$ be as derived in Proposition 2.5 and denote

$$
V_{k}:=\left\|\Delta_{k} g\right\|_{\mathcal{R}}+\left|\Delta_{k} \eta^{1}\right|+\left|\Delta_{k} \eta^{2}\right|+\left|\Delta_{k} u\right| .
$$

Then there exist constants $\beta_{1}, \beta_{2}$ depending only on $C, C_{K}, C_{J}, K_{0}$, and $\gamma$ such that for every $k \in[n]$

$$
\begin{aligned}
& \quad\left|\Delta_{k}\left(\xi^{1}-\xi^{2}\right)\right|+C \Delta_{k}\left|B\left(r^{1}, x^{1}\right)-B\left(r^{2}, x^{2}\right)\right| \\
& \leq C K_{0} \gamma\left|\Delta_{k}\left(\eta^{1}-\eta^{2}\right)\right|+\beta_{1} V_{k}\left|\xi_{k-1}^{1}-\xi_{k-1}^{2}\right| \\
& \quad+\beta_{2} V_{k}\left(1+V_{k}\right)\left(\left|\eta_{i-1}^{1}-\eta_{i-1}^{2}\right|+\left|\Delta_{k}\left(\eta^{1}-\eta^{2}\right)\right|\right) .
\end{aligned}
$$

Proof. We start from Lemma 5.1. Since $u^{1}=u^{2}$ all terms concerning $u$ cancel and $x_{k}^{1}-x_{k}^{2}=\xi_{k}^{2}-\xi_{k}^{1}$. Note furthermore that

$$
\max _{i \in\{k, k-1\}}\left\|r_{i}^{1}-r_{i}^{2}\right\|_{\mathcal{R}} \leq\left\|r_{i-1}^{1}-r_{i-1}^{2}\right\|_{\mathcal{R}}+\left\|\Delta_{i}\left(r^{1}-r^{2}\right)\right\|_{\mathcal{R}} .
$$

Putting in the definition of $r^{i}$ we can simply estimate the first term of the right hand side by

$$
\left\|r_{i-1}^{1}-r_{i-1}^{2}\right\|_{\mathcal{R}} \leq\left\|g_{i-1}\left(u_{i-1}, \eta_{i-1}^{1}\right)-g_{i-1}\left(u_{i-1}, \eta_{i-1}^{2}\right)\right\|_{\mathcal{R}} \leq \gamma\left|\eta_{i-1}^{1}-\eta_{i-1}^{2}\right| .
$$

It remains to derive an estimate for $\left\|\Delta_{i}\left(r^{1}-r^{2}\right)\right\|_{\mathcal{R}}$. This is easily done by

$$
\begin{aligned}
& \left\|\Delta_{i}\left(r^{1}-r^{2}\right)\right\|_{\mathcal{R}} \\
& =\left\|g_{i}\left(u_{i}, \eta_{i}^{1}\right)-g_{i}\left(u_{i}, \eta_{i}^{2}\right)-g_{i-1}\left(u_{i-1}, \eta_{i-1}^{1}\right)+g_{i-1}\left(u_{i-1}, \eta_{i-1}^{2}\right)\right\|_{\mathcal{R}} \\
& \leq \gamma\left(\left|\eta_{i}^{1}-\eta_{i}^{2}\right|+\left|\eta_{i-1}^{1}-\eta_{i-1}^{2}\right|\right) \\
& \leq \gamma\left|\Delta_{i}\left(\eta^{1}-\eta^{2}\right)\right|+2 \gamma\left|\eta_{i-1}^{1}-\eta_{i-1}^{2}\right| .
\end{aligned}
$$

Now plugging all of this into Lemma 5.1 we obtain the estimate.

Theorem 5.3 (Existence and uniqueness for very small jumps). Assume that Hypothesis 2.4 holds. Furthermore let

$$
\delta:=C K_{0} \gamma<1
$$

and $u \in B V_{L}^{c_{u}}(0, T ; X), g \in B V_{L}^{c_{g}}\left(0, T ; C_{\omega, \gamma}^{1}(X \times X ; \mathcal{R})\right)$ and $x_{0} \in Z(g(0, u(0)$, $\left.\left.x_{0}-u(0)\right)\right)$. Then there exists some $v>0$ such that there exits a unique solution to Problem 2.2 if $c_{g}, c_{u} \leq v$. 
Proof. If $\xi \in B V_{L}(0, T ; X)$ is a solution of the quasivariational sweeping process then

$$
\forall[r, s] \subset[0, T]: \operatorname{Var}(\xi,[r, s]) \leq S(\operatorname{Var}(g,[r, s]), \operatorname{Var}(u,[r, s])) .
$$

Here $S$ is the function defined in (3.13). Therefore let us define

$$
\Omega:=\left\{\xi \in B V_{L}^{c}(0, T ; X): \xi(0)=u(0)-x_{0} \text { and (5.10) holds }\right\} .
$$

It is yet another straightforward proof to show that $\Omega$ is closed with respect to the $B V$-norm. Furthermore for any $\eta \in \Omega$ and $t \in[0, T]$ it holds that

$$
|\eta(t+)-\eta(t)| \leq \frac{1}{1-\delta}\left(C K_{0}\|g(t+)-g(t)\|+\left(1+C K_{0} \omega\right)|u(t+)-u(t)|\right) .
$$

We define the operator $\mathcal{A}: \Omega \rightarrow B V_{L}, \mathcal{A}: \eta \mapsto \xi$, which maps any $\eta$ to the solution of the sweeping process with input $u, g(\cdot, u(\cdot), \eta(\cdot))$ and $x_{0}$. Our aim is to prove that $\mathcal{A}$ is a contraction on $\Omega$ with respect to a weighted $B V$-norm. First note that $\mathcal{A}(\Omega) \subset \Omega$. Now choose $\eta^{1}, \eta^{2} \in \Omega$ and $\varepsilon>0$. Let $\left(t_{n}^{f}\right)_{n=0}^{N_{f}}$ be the corresponding approximating partition as constructed in Lemma 4.2 of $f \in\{u, g\}$. Furthermore choose a partition of $\left(t^{i}\right)_{n=1}^{N_{i}}$ be a partition of $[0, T]$ such that $t_{0}=0$, $t_{n}=N$ and

$$
\forall n \in\left[N_{i}\right]: \forall s, t \in\left(t_{n-1}^{i}, t_{n}^{i}\right]:\left|\eta^{i}(s)-\eta^{i}(t)\right| \leq \varepsilon
$$

Let $\left(\widehat{t_{n}}\right)_{n=0}^{\widehat{N}} \in \mathcal{D}_{[0, T]}$ contain all above partitions, that is

$$
\left\{\widehat{t_{n}}: n \in[\widehat{N}] \cup\{0\}\right\} \supset\left\{t_{n}^{r}: r \in\{f, g, 1,2\} \wedge n \in\left[N_{r}\right] \cup\{0\}\right\} .
$$

We now construct the approximating step functions $\widehat{f}$ for $f \in\left\{u, g, \eta^{1}, \eta^{2}\right\}$. To not overload the notation we drop the dependence on $\varepsilon$. We choose the sequence $\left(t_{n}\right)_{n=1}^{N}$ such that $N:=2 \widehat{N}$ and

$$
t_{n}=\widehat{t}_{n / 2} \text { for } n \text { even and } t_{n}=\frac{1}{2}\left(\widehat{t}_{(n-1) / 2}+\widehat{t}_{(n+1) / 2}\right) \text { for } n \text { odd. }
$$

Now define $\widehat{f}$ by

$$
\widehat{f_{k}}= \begin{cases}f\left(\widehat{t}_{k} / 2\right) & \text { for } k \text { even } \\ f\left(\widehat{t}_{(k-1) / 2}+\right) & \text { for } k \text { odd }\end{cases}
$$

Let $\widehat{\xi}^{i}$ be the corresponding solutions of the sweeping process with input functions $\widehat{g}, \widehat{u}, \widehat{\eta}^{i}$. Due to Corollary 5.2 we have

$$
\begin{aligned}
& \left|\Delta_{k}\left(\widehat{\xi}^{1}-\widehat{\xi}^{2}\right)\right|+C \Delta_{k}\left|B\left(\widehat{r}^{1}, \widehat{x}^{1}\right)-B\left(\widehat{r}^{2}, \widehat{x}^{2}\right)\right| \\
& \leq\left(C K_{0} \gamma+\beta_{2} V_{k}\left(1+V_{k}\right)\right)\left|\Delta_{k}\left(\widehat{\eta}^{1}+\widehat{\eta}^{2}\right)\right| \\
& \quad+\beta_{1} V_{k}\left|\widehat{\xi}_{k-1}^{1}-\widehat{\xi}_{k-1}^{2}\right|+\beta_{2} V_{k}\left(1+V_{k}\right)\left|\widehat{\eta}_{i-1}^{1}-\widehat{\eta}_{i-1}^{2}\right|,
\end{aligned}
$$


where $V_{k}=\left|\Delta_{k} \widehat{u}\right|+\left|\Delta_{k} \widehat{g}\right|+\left|\Delta_{k} \widehat{\eta}^{1}\right|+\left|\Delta_{k} \widehat{\eta}^{2}\right|$. For $k \geq 1$ odd we get

$$
\begin{aligned}
\left|\Delta_{k} \widehat{\eta}^{i}\right| & =\left|\eta^{i}\left(\widehat{t}_{(k-1) / 2}\right)-\eta^{i}\left(\widehat{t}_{(k-1) / 2}+\right)\right| \\
& \leq S\left(\left|g\left(\widehat{t}_{(k-1) / 2}+\right)-g\left(\widehat{t}_{(k-1) / 2}\right)\right|,\left|u\left(\widehat{t}_{(k-1) / 2}+\right)-u\left(\widehat{t}_{(k-1) / 2}\right)\right|\right) \\
& \leq S\left(c_{g}, c_{u}\right) .
\end{aligned}
$$

If $k$ is even we have

$$
\begin{aligned}
\left|\Delta_{k} \widehat{\eta}^{i}\right| & =\left|\eta^{i}\left(\widehat{t}_{k / 2}\right)-\eta^{i}\left(\widehat{t}_{(k-1) / 2}+\right)\right| \\
& \left.\leq S\left(\operatorname{Var}\left(g,\left(t_{(k-1) / 2}, t_{k / 2}\right]\right), \operatorname{Var}\left(u,\left(t_{(k-1) / 2}, t_{k / 2}\right]\right)\right)\right) .
\end{aligned}
$$

Thus we estimate $V_{k} \leq \widetilde{V}_{k}$ with

$$
\widetilde{V}_{k}=\left|\nabla_{k} \widehat{g}\right|+\left|\nabla_{k} \widehat{u}\right|+ \begin{cases}S\left(\mid g \widehat{t}_{(k-1) / 2}+\right)-g\left(\widehat{t}_{(k-1) / 2}\right) \mid, & \\ \left.\left|u\left(\widehat{t}_{(k-1) / 2}+\right)-u\left(\widehat{t}_{(k-1) / 2}\right)\right|\right) & \text { for } k \text { odd } \\ & \\ & \left(\operatorname{Var}\left(g,\left(t_{(k-1) / 2}, t_{k / 2}\right]\right),\right. \\ \left.\left.\operatorname{Var}\left(u,\left(t_{(k-1) / 2}, t_{k / 2}\right]\right)\right)\right) & \text { for } k \text { even. }\end{cases}
$$

Assume that $c_{g}, c_{u} \leq v$ and choose $\varepsilon \leq \frac{1}{4} v$. Then by means of (4.25) we have

$$
\left.\operatorname{Var}\left(g,\left(t_{(k-1) / 2}, t_{k / 2}\right]\right) \leq v \text { and } \operatorname{Var}\left(u,\left(t_{(k-1) / 2}, t_{k / 2}\right]\right)\right) \leq v .
$$

Now we choose $v$ such that

$$
\delta+\beta_{2} 2(v+S(v, v))(1+2(v+S(v, v)))=: \delta^{\prime}<1 .
$$

Then $\left(C K_{0} \gamma+\beta_{2} \widetilde{V}_{k}\left(1+\widetilde{V}_{k}\right)\right) \leq \delta^{\prime}$ and applying Lemma A.1 we get

$$
\sum_{k=1}^{N}\left|\Delta_{k}\left(\widehat{\xi}^{1}-\widehat{\xi}^{2}\right)\right| w_{k} \leq \rho \sum_{k=1}^{N}\left|\Delta_{k}\left(\widehat{\eta}^{1}-\widehat{\eta}^{2}\right)\right| w_{k}
$$

for some $\rho<1$ and

$$
w_{k}=\exp \left\{-\frac{1}{\vartheta} \sum_{i=1}^{k} \widetilde{V}_{i}\right\}
$$

with $\vartheta$ small enough. For the given $\varepsilon$ and $\left(t_{k}\right)_{k=1}^{N}$ define $V_{f}^{\varepsilon}:[0, T] \rightarrow \mathbb{R}$ by

$$
V_{f}^{\varepsilon}: t \mapsto \begin{cases}\operatorname{Var}\left(f,\left[0, t_{k-1}\right]\right)+\left|f\left(t_{k-1}\right)-f\left(t_{k-1}+\right)\right| & \text { for } t \in\left(t_{k-1}, t_{k}\right], k \text { odd } \\ \operatorname{Var}\left(f,\left[0, t_{k}\right]\right) & \text { for } t \in\left(t_{k-1}, t_{k}\right], k \text { even }\end{cases}
$$

Then (5.20) can be rewritten into

$$
\operatorname{Var}_{\widehat{w}}\left(\widehat{\xi}^{1}-\widehat{\xi}^{2}\right) \leq \rho \operatorname{Var}_{\widehat{w}}\left(\widehat{\eta}^{1}-\widehat{\eta}^{2}\right)
$$


where $\widehat{w}:=\exp (-(1 / \delta) \widetilde{V}(t))$ and $\widetilde{V}:[0, T] \rightarrow \mathbb{R}_{>0}^{+}$is defined by

$$
\widetilde{V}(t)=\operatorname{Var}(\widehat{g},[0, t])+\operatorname{Var}(\widehat{u},[0, t])+S\left(V_{g}^{\varepsilon}(t), V_{u}^{\varepsilon}(t)\right) .
$$

Letting $\varepsilon \rightarrow 0$ we have

$$
\widehat{\eta}^{i} \stackrel{\|\cdot\|_{\infty}}{\rightarrow} \eta^{i}, \widehat{g} \stackrel{\|\cdot\|_{\infty}}{\rightarrow} g \text { and } \widehat{u} \stackrel{\|\cdot\|_{\infty}}{\rightarrow} u
$$

and consequently also $\widehat{\xi}^{i} \stackrel{\|\cdot\|_{\infty}}{\rightarrow} \xi$. We now prove that

$$
\widetilde{V} \stackrel{\|\cdot\|_{\infty}}{\rightarrow} V
$$

where $V$ is defined by

$$
V(t)=\operatorname{Var}(g,[0, t])+\operatorname{Var}(u,[0, t])+S(\operatorname{Var}(g,[0, t]), \operatorname{Var}(u,[0, t])) .
$$

First remember that $\operatorname{Var}(\widehat{f},[0, \cdot]) \stackrel{\|\cdot\|_{\infty}}{\rightarrow} \operatorname{Var}(f,[0, \cdot])$ for $f \in\{g, u\}$ by the choice of the approximating sequence. Due to the construction of $f$ we have for $t \in$ $\left(t_{k-1}, t_{k}\right)$ that $\operatorname{Var}(\widehat{f},[0, t])=\operatorname{Var}\left(\widehat{f},\left[0, t_{k}\right]\right)$ if $k$ is odd and $\operatorname{Var}(\widehat{f},[0, t])=$ $\operatorname{Var}\left(\widehat{f},\left[0, t_{k-1}\right]\right)+\left|f\left(t_{k-1}+\right)-f\left(t_{k-1}\right)\right|$ for $k$ even. Hence we can estimate

$$
\left\|\operatorname{Var}(\widehat{f},[0, t])-V_{f}^{\varepsilon}(t)\right\|_{\infty} \leq 2 \varepsilon
$$

and deduce $V_{f}^{\varepsilon} \stackrel{\|\cdot\|_{\infty}}{\rightarrow} \operatorname{Var}(f,[0, \cdot))$. Since $S(\cdot, \cdot)$ is nothing but a sum of two linear terms this implies (5.26). Therefore we have

$$
\widehat{w} \stackrel{\|\cdot\|_{\infty}}{\rightarrow} w=\exp \{-(1 / \delta) V(t)\}
$$

and due to Proposition 4.7 we obtain

$$
\operatorname{Var}_{w}\left(\xi^{1}-\xi^{2}\right) \leq \liminf _{\varepsilon} \operatorname{Var}_{\widehat{w}}\left(\widehat{\xi}^{1}-\widehat{\xi}^{2}\right)
$$

To estimate the right hand side we use

$$
\begin{aligned}
& \limsup _{\varepsilon}\left(\operatorname{Var}_{\widehat{w}}\left(\hat{\eta}^{1}-\widehat{\eta}^{2}\right)-\operatorname{Var}_{w}\left(\eta^{1}-\eta^{2}\right)\right) \\
& \leq \underset{\varepsilon}{\limsup }\left(\operatorname{Var}_{w}\left(\widehat{\eta}^{1}-\widehat{\eta}^{2}\right)-\operatorname{Var}_{w}\left(\eta^{1}-\eta^{2}\right)\right) \\
& +\underset{\varepsilon}{\limsup }\left(\operatorname{Var}_{\widehat{w}}\left(\widehat{\eta}^{1}-\widehat{\eta}^{2}\right)-\operatorname{Var}_{w}\left(\widehat{\eta}^{1}-\widehat{\eta}^{2}\right)\right) .
\end{aligned}
$$

The second term tends to zero as a consequence of (4.37). Due to the choice of $\widehat{\eta}^{i}$ we can calculate

$$
\operatorname{Var}_{w}\left(\widehat{\eta}^{1}-\widehat{\eta}^{2}\right)=\sum_{k=1}^{N} w\left(t_{k-1}+\right)\left|\widehat{\eta}^{1}\left(t_{k}\right)-\widehat{\eta}^{2}\left(t_{k}\right)-\left(\widehat{\eta}^{1}\left(t_{k-1}\right)-\widehat{\eta}^{2}\left(t_{k-1}\right)\right)\right| .
$$


For the sake of readability let us denote $\eta^{1}-\eta^{2}$ by $\delta \eta$. If $k$ is odd we have

$$
\left|\widehat{\eta}^{1}\left(t_{k}\right)-\widehat{\eta}^{2}\left(t_{k}\right)-\left(\widehat{\eta}^{1}\left(t_{k-1}\right)-\widehat{\eta}^{2}\left(t_{k-1}\right)\right)\right|=\left|\delta \eta\left(t_{k-1}+\right)-\delta \eta^{2}\left(t_{k-1}\right)\right|
$$

and if $k$ is even we can evaluate that term to

$$
\left|\widehat{\eta}^{1}\left(t_{k}\right)-\widehat{\eta}^{2}\left(t_{k}\right)-\left(\widehat{\eta}^{1}\left(t_{k-1}\right)-\widehat{\eta}^{2}\left(t_{k-1}\right)\right)\right|=\left|\delta \eta\left(t_{k}\right)-\delta \eta\left(t_{k-2}+\right)\right| .
$$

Furthermore we can bound the weighted total variation of $\xi^{1}-\xi^{2}$ from below by

$$
\begin{aligned}
& \operatorname{Var}_{w}\left(\xi^{1}-\xi^{2}\right) \\
& \quad \sum_{\{k \in[N]: k=2 r-1, r \in \mathbb{N}\}} w\left(t_{k-1}+\right)\left|\delta \eta\left(t_{k-1}+\right)-\delta \eta\left(t_{k-1}\right)\right| \\
& \quad+\sum_{\{k \in[N]: k=2 r, r \in \mathbb{N}\}} w\left(t_{k-1}+\right)\left|\delta \eta\left(t_{k-2}+\right)-\delta \eta\left(t_{k-1}+\right)\right| \\
& \quad+\sum_{\{k \in[N]: k=2 r, r \in \mathbb{N}\}} w\left(t_{k}\right)\left|\delta \eta\left(t_{k-1}+\right)-\delta \eta\left(t_{k}\right)\right| .
\end{aligned}
$$

Therefore we can estimate

$$
\begin{aligned}
& \operatorname{Var}_{w}\left(\widehat{\eta}^{1}-\widehat{\eta}^{2}\right)-\operatorname{Var}_{w}\left(\eta^{1}-\eta^{2}\right) \\
& \leq \sum_{\{k \in[N]: k=2 r, r \in \mathbb{N}\}}\left(w\left(t_{k-1}+\right)-w\left(t_{k}\right)\right)\left|\delta \eta\left(t_{k-1}+\right)-\delta \eta\left(t_{k}\right)\right| \\
& \leq \varepsilon(w(T)-w(0)),
\end{aligned}
$$

and finally obtain

$$
\operatorname{Var}_{w}\left(\xi^{1}-\xi^{2}\right) \leq \rho \operatorname{Var}_{w}\left(\eta^{1}-\eta^{2}\right) .
$$

This indeed is the contraction property and the Banach fixed point theorem grants the existence of a unique solution to Problem 2.2 within the set $\Omega$.

By simple translation one can proof Theorem 5.3 on any time interval $[r, s]$ with $-\infty<r<s<\infty$. Under the above conditions we denote the corresponding solution operator $\mathcal{S}$, that is

$$
\mathcal{S}\left(x_{0}-u(r), u, g,[r, s]\right)=\xi
$$

where $\xi$ is the unique solution of the quasivariational sweeping process with initial value $x_{0}$ and input $u, g$ on the time interval $[r, s]$. 


\subsection{Construction of a solution}

In this part we are going to construct a solution $\xi$ to the quasivariational sweeping process. We first need the following result.

Proposition 5.4. Let $u \in B V_{L}(0, T ; X)$ then for each $\varepsilon>0$ there exists an $N=$ $N(\varepsilon, \operatorname{Var}(u)) \in \mathbb{N}$ such that

$$
\#\{t \in[0, T):|u(t)-u(t+)| \geq \varepsilon\} \leq N
$$

Now we can prove

Proposition 5.5. Assume that Hypothesis 2.4, (2.9) and (2.10) are satisfied. Then there exists a solution to the quasivariational sweeping process.

Proof. Choose $v$ such that (5.19) is satisfied. By Proposition 5.4 we can choose $\left(t_{n}\right)_{n=0}^{N} \in \mathcal{D}_{[0, T]}$ such that $t_{0}=0, t_{N}=T$ and

$$
\forall n \in[N]: \forall t \in\left(t_{n-1}, t_{n}\right):|u(t)-u(t+)| \leq v \wedge|g(t)-g(t+)| \leq v .
$$

For any $f \in G(0, T ; \mathcal{Y})$ and each $n \in[N]$ we denote by

$$
\widehat{f}^{n}: \begin{cases}{\left[t_{n-1}, t_{n}\right]} & \rightarrow \mathcal{Y} \\ t & \mapsto \begin{cases}f(t) & \text { for } t \in\left(t_{n-1}, t_{n}\right] \\ f\left(t_{n-1}+\right) & \text { for } t=t_{n-1}\end{cases} \end{cases}
$$

a restriction of $f$ to $\left[t_{n-1}, t_{n}\right]$ with no jump at its initial time. We set $\xi_{0}=u(0)-x_{0}$ and $k=1$. While $k \leq N$ do

(1) $\bar{\xi}_{k-1}:=\mathcal{E}\left(\xi_{k-1}, u\left(t_{k-1}\right), g\left(t_{k-1}\right), u\left(t_{k-1}+\right), g\left(t_{k-1}+\right)\right)$

(2) $\widehat{\xi}^{k}:=\mathcal{S}\left(\bar{\xi}_{k-1}, \widehat{u}^{k}, \widehat{g}^{k},\left[t_{k-1}, t_{k}\right]\right)$

(3) $\xi_{k}:=\widehat{\xi}^{k}\left(t_{k}\right), k:=k+1$.

We define $\xi:[0, T] \rightarrow X$ by

$$
\xi(t):=\left\{\begin{array}{ll}
\xi_{0} & \text { if } t=0 \\
\xi^{n}(t) & \text { if } t \in\left(t_{n-1}, t_{n}\right]
\end{array} .\right.
$$

It remains to prove that $\xi$ is a solution to Problem 2.2. First we note that $\xi \in$ $B V_{L}(0, T ; X)$. This is due to

$$
\operatorname{Var}(\xi) \leq \sum_{k=1}^{N}\left(\operatorname{Var}\left(\widehat{\xi}^{k},\left[t_{k-1}, t_{k}\right]\right)+\left|\xi_{k-1}-\bar{\xi}_{k-1}\right|\right)
$$

and the left-continuity of $\widehat{\xi}^{k}$. Due to (3.11) we have for all $n \in[N]$

$$
\xi\left(t_{n-1}+\right)=\widehat{\xi}^{n}\left(t_{n-1}+\right)=\widehat{\xi}^{n}\left(t_{n-1}\right)=\bar{\xi}_{n-1} .
$$


For $z \in \mathcal{T}(\xi)$ we compute

$$
\begin{aligned}
& \int_{0}^{T}\langle u(t)-\xi(t)-z(t), d \xi(t)\rangle \\
& =\sum_{n=1}^{N}\left(\int_{0}^{T}\left\langle(u(t+)-\xi(t+)-z(t)) \chi_{\left\{t_{n-1}\right\}}(t), d \xi(t)\right\rangle\right. \\
& \left.\quad+\int_{0}^{T}\left\langle(u(t+)-\xi(t+)-z(t)) \chi_{\left(t_{n-1}, t_{n}\right)}(t), d \xi(t)\right\rangle\right) .
\end{aligned}
$$

The first term within can be evaluated by

$$
\begin{aligned}
& \int_{0}^{T}\left\langle(u(t+)-\xi(t+)-z(t)) \chi_{\left\{t_{n-1}\right\}}(t), d \xi(t)\right\rangle \\
& =\left\langle u\left(t_{n-1}+\right)-\xi\left(t_{n-1}+\right)-z\left(t_{n-1}\right), \xi\left(t_{n-1}+\right)-\xi\left(t_{n-1}\right)\right\rangle \geq 0 .
\end{aligned}
$$

Remember that $z\left(t_{n-1}\right) \in Z\left(g\left(t_{n-1}+, u\left(t_{n-1}+\right), \xi\left(t_{n-1}+\right)\right)\right)$. The inequality is due to (5.43) and the definition of $\bar{\xi}_{n-1}$. For the second term we make use of Lemma B.1 to see that

$$
\begin{aligned}
& \int_{0}^{T}\left\langle(u(t+)-\xi(t+)-z(t)) \chi_{\left(t_{n-1}, t_{n}\right)}(t), d \xi(t)\right\rangle \\
& =\int_{t_{n-1}}^{t_{n}}\left\langle\left(\widehat{u}^{n}(t+)-\widehat{\xi}^{n}(t+)-z(t)\right) \chi_{\left(t_{n-1}, t_{n}\right)}(t), d \widehat{\xi}^{n}(t)\right\rangle \geq 0 .
\end{aligned}
$$

Here the inequality is a consequence of the fact that $\widehat{\xi}^{n}$ is by definition a solution of the quasivariational sweeping process on the interval $\left[t_{n-1}, t_{n}\right]$. Thus we have for any $z \in \mathcal{T}(\xi)$ that

$$
\int_{0}^{T}\langle u(t)-\xi(t)-z(t), d \xi(t)\rangle \geq 0
$$

and the proof is complete.

\subsection{Uniqueness of the solution}

We show that the solution constructed above is indeed a unique solution. Before let us state the following.

Lemma 5.6. Let $\xi \in B V_{L}(0, T ; X)$ be a solution of the quasivariational sweeping process on $[0, T]$. Choose any $[r, s] \subset[0, T]$ and define for $f \in\{g, u, \xi\} \widehat{f}:$ $[r, s] \rightarrow \mathcal{Y}$ (where $\mathcal{Y}$ is the appropriate space) by

$$
\widehat{f}: t \mapsto \begin{cases}f(r+) & \text { if } t=r \\ f(t) & \text { else. }\end{cases}
$$

Then $\widehat{\xi}$ solves the quasivariational sweeping process on $[r, s]$ with input functions $\widehat{g}, \widehat{u}$ and initial value $\xi(r+)$. 
Proof. Obviously $\widehat{\xi}(t)$ has the desired initial value. Choose any $\widehat{z} \in G(r, s ; X)$ such that $\widehat{z}(t) \in Z(g(t+, u(t+), \xi(t+)))$. Define

$$
z(t):=(u(t+)-\xi(t+)) \chi_{[0, T] \backslash(r, s)}(t)+\widehat{z}(t) \chi_{(r, s)}(t) .
$$

Then due to Lemma B.1 - note that $\xi$ is left continuous - we have

$$
0 \leq \int_{0}^{T}\langle u(t+)-\xi(t+)-z(t), d \xi(t)\rangle=\int_{r}^{s}\langle u(t+)-\widehat{\xi}(t+)-\widehat{z}(t), d \widehat{z}(t)\rangle .
$$

Thus $\widehat{\xi}$ satisfies the quasi-variational inequality and the proof is complete.

Proposition 5.7. Assume that Hypothesis 2.4 holds. Furthermore let (2.9) and (2.10) be fullfilled. Then the solution to Problem 2.2 is unique.

Proof. Let $\left(t_{n}\right)_{n=0}^{N}$ be the partition of [0,T] corresponding to (5.39). Assume that $\xi, \eta \in B V_{L}(0, T ; X)$ solve Problem 2.2 with $\eta \neq \xi$, that is there exists some $t \in[0, T]$ such that $\xi(t) \neq \eta(t)$. Let

$$
\tau:=\inf \{t \in[0, T]: \xi(t) \neq \eta(t)\} .
$$

We make the convention that $\tau=T$ if $\xi \equiv \eta$. This is motivated by the fact that $\xi(\tau)=\eta(\tau)$. If $\tau=0$ it is immediate, otherwise it is a consequence of the left continuity. We first show that if $\tau \in\left(t_{n-1}, t_{n}\right]$, then $\tau=t_{n}$. First notice $\xi(t)=\eta(t)$ for all $t \in\left(t_{n-1}, \tau\right]$ and especially $\xi\left(t_{n-1}+\right)=\eta\left(t_{n-1}+\right)$. Due to Lemma 5.6 both $\widehat{\xi}^{n}$ and $\widehat{\eta}^{n}$ solve the quasivariational evolution equation with initial value $\xi\left(t_{n-1}+\right)$. By Theorem 5.3 we know the solution to be unique, in other words

$$
\widehat{\xi}^{n}=\mathcal{S}\left(\xi\left(t_{n-1}+\right), \widehat{u}^{n}, \widehat{g}^{n},\left[t_{n-1}, t_{n}\right]\right)=\widehat{\eta}^{n} .
$$

Therefore $\xi(t)=\eta(t)$ for all $t \in\left(t_{n-1}, t_{n}\right]$ and we can safely assume that $\tau=t_{n}$ for some $n \in[N] \cup 0$. If $n=N$ we already have $\xi \equiv \eta$. Otherwise by testing inequality (2.4) with

$$
z(t)=(u(t+)-\xi(t+)) \chi_{[0, T] \backslash\left\{t_{n}\right\}}(t)+y \chi_{\left\{t_{n}\right\}}(t)
$$

for any $y \in Z\left(g\left(t_{n}+, u\left(t_{n}+\right), g\left(t_{n}+\right)\right)\right.$ and the respective function for $\eta$ we derive that

$$
\xi\left(t_{n}+\right)=\mathcal{E}\left(\xi\left(t_{n}\right), u\left(t_{n}\right), g\left(t_{n}\right), u\left(t_{n}+\right), g\left(t_{n}+\right)\right)=\eta\left(t_{n}+\right) .
$$

Due to Lemma 5.6 we know that $\widehat{\xi}^{n+1}$ and $\widehat{\eta}^{n+1}$ solve the quasivariational evolution equation on $\left[t_{n}, t_{n+1}\right]$ with initial value $\xi\left(t_{n}+\right)$. Since its solution is unique we know that $\xi(t)=\eta(t)$ for all $t \leq t_{n+1}$, hence $\tau \geq t_{n+1}$ - a contradiction. Therefore $\tau=t_{N}=T$ and $\xi \equiv \eta$.

Theorem 2.6 is now the immediate consequence of Propositions 5.5 and 5.7. 


\section{On the jump size condition}

In this section we discuss condition (2.10) restricting the jump size in Theorem 2.6 and whether it is necessary. We start by showing that (2.9) alone is not sufficient to guarantee uniqueness. However in some cases (2.10) is not necessary for which we will give two examples. It suffices to consider Problem 3.5 which is the critical part. If we can guarantee a unique solution for it, we can guarantee a unique solution for Problem 2.2 as well.

\subsection{Necessity of a jump size condition for general convex sets}

We give an example of nonuniqueness of the quasivariational inequality for $C K_{0} \gamma$ arbitrarily small. Thus the condition (2.9) is not sufficient and an additional one has to be chosen.

Example 6.1. Choose $f \in C^{\infty}(\mathbb{R})$ such $f(x)=x$ in $[-1 / 2,1 / 2],\|f\|_{\infty} \leq 1$ and $\left\|f^{\prime}\right\|_{\infty} \leq 1$. We furthermore choose the spaces $X=\mathbb{R}^{2}$ with $\mathcal{R}=\mathbb{R}$. Consider polyhedra of the following type

$$
Z(r)=\left\{x \in \mathbb{R}^{2}: A(r) x \leq w(r)\right\}
$$

with

$$
A(r)=\left(\begin{array}{rr}
1 & 0 \\
-1 & 0 \\
0 & -1 \\
f(r) & l
\end{array}\right), w(r)=\left(\begin{array}{c}
1 \\
1 \\
1 \\
1-f(r)^{2}
\end{array}\right) .
$$

The real number $l>1$ remains to be chosen. Note that for $l>1$ we have $0 \in Z(r)$ and we can calculate the Minkowski functional of $Z(r)$ by

$$
M(r, x)=\max \left\{\frac{a_{i} \cdot x}{w_{i}}, i \in[4]\right\} .
$$

Here $a_{i}$ denotes the $i$-th row of $A$. Therefore it is easy to compute that

$$
\partial_{r} M(r, x)=\frac{\left\langle\left(\begin{array}{c}
f^{\prime}(r) \\
0
\end{array}\right), x\right\rangle}{\left(l-f(r)^{2}\right)}-2 \frac{\left\langle\left(\begin{array}{c}
f(r) \\
l
\end{array}\right), x\right\rangle f(r) f^{\prime}(r)}{\left(l-f(r)^{2}\right)^{2}}
$$

if $4 \in \operatorname{argmax}\left\{\frac{a_{i} \cdot x}{w_{i}}, i \in[4]\right\}$ and 0 otherwise. Note that for $l \geq 2$ we have

$$
1 / 4\left|a_{4}(r)\right|^{2}=1 / 4 f(r)^{2}+1 / 4 l^{2} \leq 1 / 2 l^{2} \leq\left(l-f(r)^{2}\right)^{2}
$$

and $B_{1 / 2}(0) \subset K(r)$. Furthermore we see that $B_{2}(0) \supset K(r)$. Thus we can set $C=2$. Using [2, Lemma 3.1] we have $M(r, x) \leq 2|x|$ for any $x \in \mathbb{R}^{2}$ and 
therefore $M(r, x) \leq 4$ for all $x \in B_{C}(0)$. Therefore and by our above calculations we can estimate

$$
|K(r, x)| \leq c \frac{1}{l-1}
$$

where $c$ is a suitable constant. Setting $g: \mathbb{R}^{2} \times \mathbb{R}^{2} \rightarrow \mathbb{R},(u, \xi) \mapsto u-\xi$ we have $\gamma=1$. Thus we finally obtain

$$
\delta=C K_{0} \gamma \leq c \frac{1}{l-1} .
$$

Choosing $l$ arbitrary large we can make $\delta$ arbitrary small. However setting $x^{0}=$ $(0,1), u^{0}=(0,0), u^{1}=(0, l)$ we have that any $x^{1}=(z, 1)$ with $z \in[-1 / 2,1 / 2]$ is a solution to

$$
x_{1}=Q_{Z\left(g\left(u^{1}, u^{1}-x^{1}\right)\right)}\left(x^{0}+\Delta u\right) .
$$

Note that the set $Z(r)$ a polyhedron and thus does not satisfy Hypothesis 2.4. However by smoothing the edges (let's say for $\left|x_{1}\right|>3 / 4$ ) there is no essential change in the behavior and the aforementioned conditions are satisfied.

We see that the situation is rather unpleasant as a condition on the sizes of jumps is in general needed. However we will give some examples where it can be omitted through using additional information on the convex sets.

\subsection{Balls with variable radius}

This is perhaps the simplest example. Let $\mathcal{R}=\mathbb{R}$ and assume that

$$
Z(r)=B_{r}(0)
$$

where $B_{r}(0)=\{x \in X:|x| \leq r\}$ is the closed ball with radius $r$. Furthermore we set

$$
g: X \rightarrow \mathbb{R}^{+}:|g(x)-g(y)|<|x-y| \forall x, y \in X, x \neq y .
$$

Proposition 6.2. For any $y \in X$ there exists a unique solution to the problem: Find छsuch that

$$
\xi=Q_{Z(g(\xi))}(y) .
$$

Proof. For uniqueness assume that both $\xi, \eta$ solve the above problem. Without loss of generality let $|\xi| \leq|\eta|$. It is straightforward to see that

$$
\xi=\frac{y}{|y|}|\xi| \text { and } \eta=\frac{y}{|y|}|\eta|
$$

with $|\xi|,|\eta| \leq|y|$. Therefore if $\xi \neq \eta$, then $|\xi|<|\eta| \leq|y|$. In this case $Q_{K(g(\xi))}(y) \neq y$ and we immediately derive $|\xi|=g(\xi)$. Furthermore by definition $|\eta| \leq g(\eta)$. We then derive

$$
|\xi-\eta| \leq|\eta|-|\xi| \leq g(\eta)-g(\xi)<|\eta-\xi|
$$


a contradiction. To prove existence denote by $Y=\{\lambda y: 1 \geq \lambda \geq 0\}$ the line between 0 and $y$. Let $\mathcal{A}: X \rightarrow Y$ be the continuous operator which maps $z \mapsto$ $Q_{Z(g(z))}(y)$. Since $Y$ is compact by the Schauder fixed point theorem $\mathcal{A}$ has a fixed point $\xi \in Y$, which solves (6.11).

Things work out so smoothly since the problem reduces to a problem on the real line. In fact by a similar method one can show that the implicit sweeping process on the real line always has a unique solution for $B V$ input functions provided that $C K_{0} \gamma<1$.

\subsection{Uniformly curved boundaries}

Assume that for all $r \in \mathcal{R}$ there exists some $\vartheta>0$ such that

$$
|n(r, x)-n(r, y)| \geq \vartheta|x-y| \forall x, y \in \partial Z(r) .
$$

In other words what we are asking for is that the curvature of the boundary is uniformly bounded from below for all $r$. With this we can sharpen the condition (2.9) such that a restriction on the jump size is no longer necessary.

Proposition 6.3. Let Hypothesis 2.4 and (6.14) hold. If

$$
\left(C K_{0}+\vartheta^{-1} C C_{J}\left(C K_{0}+2\right)\right) \gamma<1
$$

then there exists a unique solution to Problem 3.5.

Proof. The proof relies on a careful examination of the proof of Proposition 3.2 which was given in [11]. For $r, s \in \mathbb{R}$ let $Q_{r / s}$ denote the orthogonal projection onto $Z(r)$ and $Z(s)$ respectively. Our aim is to prove that

$$
\left|Q_{r}(x)-Q_{s}(x)\right| \leq\left(C K_{0}+\vartheta^{-1} C C_{J}\left(C K_{0}+2\right)\right)\|r-s\|_{\mathcal{R}} .
$$

If $x \in Z(s)$ the left hand side can be estimated from above by the Hausdorff distance $d_{H}(r, s)$. Lemma 3.3 then grants the claim. The same holds for $x \in Z(r)$. If $x \notin Z(s) \cup Z(s)$ we use equation (3.26) of the aforementioned paper where it was shown that

$$
\left|n\left(r, Q_{r}(x)\right)-n\left(s, Q_{s}(x)\right)\right| \leq\left|n\left(r, Q_{r}(x)\right)-n\left(s, Q_{s}\left(Q_{r}(x)\right)\right)\right| .
$$

Due to Proposition 3.3 we can estimate the right hand side from above by

$$
\left|n\left(r, Q_{r}(x)\right)-n\left(s, Q_{s}\left(Q_{r}(x)\right)\right)\right| \leq C C_{J}\left(d_{H}(r, s)+\|r-s\|_{\mathcal{R}}\right) .
$$

On the other hand the left hand side can be estimated from below by

$$
\begin{aligned}
& \left|n\left(r, Q_{r}(x)\right)-n\left(s, Q_{s}(x)\right)\right| \\
& \geq\left|n\left(r, Q_{r}(x)\right)-\frac{J\left(r, Q_{s}(x)\right)}{\left|J\left(r, Q_{s}(x)\right)\right|}\right|-\left|\frac{J\left(r, Q_{s}(x)\right)}{\left|J\left(r, Q_{s}(x)\right)\right|}-\frac{J\left(s, Q_{s}(x)\right)}{\left|J\left(s, Q_{s}(x)\right)\right|}\right| .
\end{aligned}
$$


Note that the outer normal is only defined at the boundary but the term $\frac{J(r, \cdot)}{|J(r, \cdot)|}$ is defined everywhere in $X$, coincides with the outer normal on the boundary and is 0 -homogeneous. For $v=1 / M\left(r, Q_{s}(x)\right)$ we have $v Q_{s}(x) \in \partial Z(r)$. Therefore we can estimate

$$
\begin{aligned}
& \left|n\left(r, Q_{r}(x)\right)-\frac{J\left(r, Q_{s}(x)\right)}{\left|J\left(r, Q_{s}(x)\right)\right|}\right| \\
& =\left|n\left(r, Q_{r}(x)\right)-n\left(r, v Q_{s}(x)\right)\right| \\
& \geq \vartheta\left|Q_{r}(x)-Q_{s}(x)\right|-\vartheta \frac{\left|Q_{s}(x)\right|}{M\left(r, Q_{s}(x)\right)}\left|M\left(r, Q_{s}(x)\right)-1\right| .
\end{aligned}
$$

Since $x \notin Z(s)$ we have $Q_{s}(x) \in \partial Z(s)$. Using [2, Lemma 3.1] we get $M\left(r, \frac{Q_{s}(x)}{\left|Q_{s}(x)\right|}\right) \geq$ $C^{-1}$. If $M\left(r, Q_{s}(x)\right) \geq 1$ then

$$
M^{2}\left(r, Q_{s}(x)\right)-M\left(s, Q_{s}(x)\right)^{2} \geq M\left(r, Q_{s}(x)\right)-1 \geq 0,
$$

if $M\left(r, Q_{s}(x)\right)<1$ then

$$
M^{2}\left(r, Q_{s}(x)\right)-M^{2}\left(s, Q_{s}(x)\right) \leq M\left(r, Q_{s}(x)\right)-1 \leq 0 .
$$

Thus we have

$$
\left|M\left(r, Q_{s}(x)\right)-1\right| \leq\left|M^{2}\left(r, Q_{s}(x)\right)-M\left(s, Q_{s}(x)\right)^{2}\right| \leq K_{0}\|r-s\|_{\mathcal{R}}
$$

and we finally obtain

$$
\begin{aligned}
& \left|n\left(r, Q_{r}(x)\right)-n\left(s, Q_{s}(x)\right)\right| \\
& \geq \vartheta\left|Q_{r}(x)-Q_{s}(x)\right|-\vartheta C K_{0}\|r-s\|_{\mathcal{R}}-C_{J} C\|r-s\|_{\mathcal{R}} .
\end{aligned}
$$

Putting all together we derive (6.16). An application of Banach's fixed point theorem now grants a unique solution to Problem 3.5.

Going through the computations we see that under the conditions of Proposition 6.3 there exists a unique solution to Problem 2.2 for all $u \in B V_{L}(0, T ; X)$, $g \in B V_{L}\left(0,, T ; C_{\omega, \gamma}^{1}(X \times X ; \mathcal{R})\right)$ and $x_{0} \in Z\left(g\left(0, u(0), u(0)-x_{0}\right)\right)$. However we refrain from doing so here and leave it to the reader.

Concluding this section we see that, if we allow for functions of bounded variation instead of only absolutely continuous functions, we pay by imposing a structural assumption in addition to the conditions of [2]. These additional assumptions can be either on the involved functions, as in the main part of the present paper, or on the convex sets, as we showed here. 


\section{Appendix}

\section{A. A discrete Gronwall-like lemma}

We prove a discrete Gronwall-like lemma adapted especially for our purposes.

Lemma A.1. Let $\left(\xi_{k}\right)_{k=0}^{N},\left(\eta_{k}\right)_{k=0}^{N} \subset X,\left(b_{k}\right)_{k=0}^{N},\left(a_{k}\right)_{k=0}^{N} \subset \mathbb{R}_{\geq 0}$ with

$$
\left|\Delta_{k} \xi\right|+\Delta_{k} b \leq a_{k}\left(\left|\xi_{k-1}\right|+\left|\eta_{k-1}\right|\right)+\delta\left|\Delta_{k} \eta\right| \quad \forall k \in[N]
$$

for some $0 \leq \delta<1$. Assume furthermore that $b_{0}=0$ and $\xi_{0}=\eta_{0}=0$. Then there exists $\varepsilon \geq 0$ and $0 \leq \rho<1$ such that for

$$
w_{k}=\exp \left\{-\frac{1}{\varepsilon} \sum_{i=1}^{k} a_{k}\right\} \quad \forall k \in[N]
$$

it holds

$$
\sum_{k=1}^{N}\left|\Delta_{k} \xi\right| w_{k} \leq \rho \sum_{k=1}^{N}\left|\Delta_{k} \eta\right| w_{k} .
$$

Proof. Choose $\varepsilon<(1-\delta) / 2$, set

$$
\rho:=\frac{\delta+\varepsilon}{1-\varepsilon}<1,
$$

and multiply both sides of (A.1) by $w_{k}$. Since $w_{k}$ is a decreasing sequence and $b_{0}=0$ we have

$$
\sum_{k=1}^{N}\left(\Delta_{k} b\right) w_{k}=b_{N} w_{N}+\sum_{k=1}^{N-1} b_{k}\left(w_{k}-w_{k-1}\right)-b_{0} w_{1} \geq 0
$$

and therefore obtain

$$
\sum_{k=1}^{N}\left|\Delta_{k} \xi\right| w_{k} \leq \sum_{k=1}^{N} a_{k} w_{k}\left(\left|\xi_{k-1}\right|+\left|\eta_{k-1}\right|\right)+\delta \sum_{k=1}^{N}\left|\Delta_{k} \eta\right| w_{k} .
$$

For the first term on the right hand side we have

$$
\begin{aligned}
\sum_{k=1}^{N} a_{k} w_{k}\left|\xi_{k-1}\right| & \stackrel{\xi_{0}=0}{\leq} \varepsilon \sum_{k=2}^{N} \frac{1}{\varepsilon} a_{k} w_{k}\left(\sum_{j=1}^{k-1}\left|\Delta_{j} \xi\right|\right) \\
& =\varepsilon \sum_{j=1}^{N-1}\left|\Delta_{j} \xi\right|\left(\sum_{k=j+1}^{N} \frac{1}{\varepsilon} a_{k} w_{k}\right) .
\end{aligned}
$$


We can estimate

$$
\sum_{k=j+1}^{N} \frac{1}{\varepsilon} a_{k} w_{k} \leq \int_{\frac{1}{\varepsilon} \sum_{k=1}^{j} a_{k}}^{\frac{1}{\varepsilon} \sum_{k=1}^{N} a_{k}} \exp (-x) \leq \exp \left\{-\frac{1}{\varepsilon} \sum_{k=1}^{j} a_{k}\right\}=w_{j}
$$

by interpreting the first term as a Riemann sum. Proceeding in the same way for $\sum a_{k} w_{k}\left|\eta_{k-1}\right|$ we obtain

$$
\sum_{k=1}^{N}\left|\Delta_{k} \xi\right| w_{k} \leq \varepsilon \sum_{k=1}^{N}\left(\left|\Delta_{k} \xi\right|+\left|\Delta_{k} \eta\right|\right) w_{k}+\delta \sum_{k=1}^{N}\left|\Delta_{k} \eta\right| w_{k} .
$$

This completes the proof.

\section{B. A lemma for the Kurzweil integral}

For a comprehensive introduction to the Kurzweil integral we point out $[8,10,14]$ and references therein. Here we prove the following technical result.

Lemma B.1. Let $u \in G(0, T ; X), \xi \in B V(0, T ; X)$ and $[r, s] \subset[0, T]$ with $r<s$. Define

$$
\widehat{\xi}:[r, s] \rightarrow X, t \mapsto \begin{cases}\xi(r+) & \text { if } t=r \\ \xi(t) & \text { if } t \in(r, s) \\ \xi(s-) & \text { if } t=s .\end{cases}
$$

Then

$$
\int_{0}^{T}\left\langle u(t) \chi_{(r, s)}(t), d \xi(t)\right\rangle=\int_{r}^{s}\langle u(t), d \widehat{\xi}(t)\rangle .
$$

Proof. It suffices to prove the above for step functions $u \in S(0, T ; X)$. In that case there exists a partition $\left(t_{n}\right)_{n=0}^{N} \in \mathcal{D}_{[0, T]}, t_{0}=r, t_{N}=s$ such that

$$
u(t) \chi_{(r, s)}(t)=\sum_{n \in[N]} u^{n} \chi_{\left(t_{n-1}, t_{n}\right)}(t)+\sum_{n \in[N-1]} \bar{u}^{n} \chi_{\left\{t_{n}\right\}}(t)
$$

for some $\left(u^{n}\right)_{n=1}^{N},\left(\bar{u}^{n}\right)_{n=1}^{N-1} \subset X$. Then we have for the left hand side integral

$$
\begin{aligned}
& \int_{0}^{T}\left\langle u(t) \chi_{(r, s)}(t), d \xi(t)\right\rangle \\
& =\sum_{n \in[N]}\left\langle u^{n}, \xi\left(t_{n}-\right)-\xi\left(t_{n-1}+\right)\right\rangle+\sum_{n \in[N-1]}\left\langle\bar{u}^{n}, \xi\left(t_{n}+\right)-\xi\left(t_{n}-\right)\right\rangle .
\end{aligned}
$$


For the right hand side we get

$$
\begin{aligned}
& \int_{r}^{s}\langle u(t), d \widehat{\xi}(t)\rangle \\
& =\sum_{n \in[N]}\left\langle u^{n}, \widehat{\xi}\left(t_{n}-\right)-\widehat{\xi}\left(t_{n-1}+\right)\right\rangle+\sum_{n \in[N-1]}\left\langle\bar{u}^{n}, \widehat{\xi}\left(t_{n}+\right)-\widehat{\xi}\left(t_{n}-\right)\right\rangle \\
& \quad+\langle u(r), \widehat{\xi}(r+)-\widehat{\xi}(r)\rangle+\langle u(s), \widehat{\xi}(s)-\widehat{\xi}(s-)\rangle .
\end{aligned}
$$

Due to the definition of $\widehat{\xi}$ the last two terms are zero and the former sums agree with the ones above. Thus for all $u \in S(0, T ; X)($ B.2) holds. Now let $\xi$ be in $G(0, T ; X)$. Then there exists a sequence of step functions $\left(\xi^{n}\right)_{n \in \mathbb{N}}$ such that $\xi^{n} \rightarrow$ $u$ with respect to $\|\cdot\|_{\infty}$. Note that then also $\widehat{\xi}^{n} \rightarrow \widehat{\xi}$ as well and the continuity of the Kurzweil integral grants the statement.

\section{References}

[1] G. Aumann, "Reelle Funktionen", Springer-Verlag, New York, 1954.

[2] M. BRokATE, P. KREJČÍ and H. SCHNABEL, On uniqueness in evolution quasivariational inequalities, J. Convex Anal. 11 (2004), 111-130.

[3] A. L. GURSOn, Continuum theory of ductile rupture by void nucleation and growth: Part I - yield criteria and flow rules for porous ductile media, J. Engrg. Mater. Tech. 99 (1977), $2-15$.

[4] V. S. Kozjankin, M. A. Krasnosel'skil and A. V. Pokrovskil̆, Vibrationally stable hysterons, Soviet Math. Dokl. 13 (1972), 1305-1309.

[5] M. A. Krasnosel'skiĬ, B. M. Darinskĭ̌, I. V. Emelin, P. P. Zabrejko, E. A. LIFshĬTS and A. V. PoKROVsKIĬ, Hysterant operator, Soviet Math. Dokl. 11 (1970), 2933.

[6] M. A. Krasnosel'skiľ and A. V. PokrovskiĬ, "Sistemy s Gisterezisom", Nauka, Moscow, 1983.

[7] P. KREJČÍ, Evolution variational inequalities and multidimensional hysteresis operators, In: "Nonlinear Differential Equations" (Chvalatice, 1998), volume 404 of Chapman \& Hall/CRC Res. Notes Math., Chapman \& Hall/CRC, Boca Raton, FL, 1999, 47-110,

[8] P. KREJČí, The Kurzweil integral with exclusion of negligible sets, Math. Bohem. 128 (2003), 277-292.

[9] P. Krejčí and P. Laurençot, Generalized variational inequalities, J. Convex Anal. 9 (2002), 159-183.

[10] P. KREJČÍ and M. Liero, Rate independent Kurzweil processes, Appl. Math. 54 (2009), $117-145$.

[11] P. KREJČÍ and T. RochE, Lipschitz continuous data dependence of sweeping processes in BV spaces, Discrete Contin. Dyn. Syst. Ser. B 15 (2011), 637-650.

[12] M. Kunze and M. D. P. Monteiro MARques, $B V$ solutions to evolution problems with time-dependent domains, Set-Valued Anal. 5 (1997), 57-72.

[13] M. Kunze and M. D. P. Monteiro MARQues, On parabolic quasi-variational inequalities and state-dependent sweeping processes, Topol. Methods Nonlinear Anal. 12 (1998), 179-191.

[14] J. KURZWEIL, Generalized ordinary differential equations and continuous dependence on a parameter, Czechoslovak Math. J. 7 (1957), 418-449. 
[15] A. MiELKE and R. Rossi, Existence and uniqueness results for a class of rate-independent hysteresis problems, Math. Models Methods Appl. Sci. 17 (2007), 81-123.

[16] A. MielKe and F. TheIL, On rate-independent hysteresis models, NoDEA Nonlinear Differential Equations Appl. 11 (2004), 151-189.

[17] M. D. P. Monteiro MARQues, Rafle par un convexe semi-continu inférieurement d'intérieur non vide en dimension finie, C. R. Acad. Sci. Paris Sér. I Math. 299 (1984), 307-310.

[18] J.-J. Moreau, Problème d'évolution associé à un convexe mobile d'un espace hilbertien, C. R. Acad. Sci. Paris Sér. A-B 276 (1973), A791-A794.

[19] J.-J. MOREAU, Evolution problem associated with a moving convex set in a Hilbert space, J. Differential Equation 26 (1977), 347-374.

[20] R. Rossi and U. STEFANELLI, An order approach to a class of quasivariational sweeping processes, Adv. Differential Equations 10 (2005), 527-552.

[21] H. Schnabel, "Zur Wohlgestelltheit des Gurson-Modells", Doctoral Thesis, Technische Universität München, 2006.

[22] U. Stefanelli, Nonlocal quasivariational evolution problems, J. Differential Equations 229 (2006), 204-228.

Department of Mathematics / M6

Technische Universität München

Boltzmannstrasse, 3

85748 Garching b. München, Germany

roche@ma.tum.de 